ASTRO-PH/0206037

Preprint typeset using $\mathrm{LAT}_{\mathrm{EX}}$ style emulateapj v. 14/09/00

\title{
THE FORMATION OF MASSIVE STARS FROM TURBULENT CORES
}

\author{
Christopher F. McKeE ${ }^{1}$ and Jonathan C. Tan ${ }^{2}$ \\ 1. Departments of Physics \& Astronomy, University of California, Berkeley, CA 94720, USA. \\ 2. Princeton University Observatory, Princeton, NJ 08544, USA. \\ Submitted 31st May 2002, Accepted 12th November 2002
}

\begin{abstract}
Observations indicate that massive stars in the Galaxy form in regions of very high surface density, $\Sigma \sim 1 \mathrm{~g} \mathrm{~cm}^{-2}$. Clusters containing massive stars and globular clusters have a column density comparable to this. The total pressure in clouds of such a column density is $P / k \sim 10^{8}-10^{9} \mathrm{~K} \mathrm{~cm}{ }^{-3}$, far greater than that in the diffuse interstellar medium or the average in giant molecular clouds. Observations show that massive star-forming regions are supersonically turbulent, and we show that the molecular cores out of which individual massive stars form are as well. The protostellar accretion rate in such a core is approximately equal to the instantaneous mass of the star divided by the free-fall time of the gas that is accreting onto the star (Stahler, Shu, \& Taam 1980). The star-formation time in this Turbulent Core model for massive star formation is several times the mean free-fall time of the core out of which the star forms, but is about equal to that of the region in which the core is embedded. The high densities in regions of massive star formation lead to typical time scales for the formation of a massive star of about $10^{5} \mathrm{yr}$. The corresponding accretion rate is high enough to overcome the radiation pressure due to the luminosity of the star. For the typical case we consider, in which the cores out of which the stars form have a density structure $\rho \propto r^{-1.5}$, the protostellar accretion rate grows with time as $\dot{m}_{*} \propto t$. We present a new calculation of the evolution of the radius of a protostar and determine the protostellar accretion luminosity. At the high accretion rates that are typical in regions of massive star formation, protostars join the main sequence at about $20 M_{\odot}$. We apply these results to predict the properties of protostars thought to be powering several observed hot molecular cores, including the Orion hot core and $\mathrm{W} 3\left(\mathrm{H}_{2} \mathrm{O}\right)$. In the Appendixes, we discuss the pressure in molecular clouds and we argue that "logatropic" models for molecular clouds are incompatible with observation.
\end{abstract}

Subject headings: hydrodynamics — ISM: clouds — stars: formation

\section{INTRODUCTION}

Massive stars are fundamental in the evolution of galaxies since they produce the heavy elements, energize the interstellar medium, and possibly regulate the rate of star formation. Remarkably little is known about how massive stars form, however: the problem is difficult observationally because massive star formation occurs in distant, highly obscured regions, and it is difficult theoretically because of the many processes that must be included. Even such a basic parameter as the time it takes to form a massive star has been uncertain. This time scale, or equivalently, the protostellar accretion rate, affects the luminosity of the protostar (particularly for masses $m_{*} \lesssim 10 M_{\odot}$ (Palla \& Stahler 1992) and the strength of protostellar outflows (Richer et al. 2000). Arguments based on extrapolating from low-mass star formation lead to formation times $t_{* f}>10^{6} \mathrm{yr}$, a significant fraction of the main-sequence lifetime of the star (Bernasconi \& Maeder 1996; McLaughlin \& Pudritz 1997, hereafter MP97; Stahler et al. 2000). Comparison with observations of hot molecular cores (Osorio, Lizano \& D'Alessio 1999; Nakano et al. 2000) suggest substantially smaller time scales, $t_{* f} \lesssim 10^{5}$ yr. An analysis based on observations of protostellar outflows suggests $t_{* f} \sim 3 \times 10^{5}$ yr (Behrend \& Maeder 2001). The small $\left(\sim 1 \times 10^{6}\right.$ yr) spread in ages of stars in the Orion Nebula Cluster (Palla \& Stahler 1999), where there is no evidence that the higher mass stars have formed systematically later compared to the lower-mass population, sets an upper limit of $t_{* f} \lesssim 1$ Myr in this case. What has been lacking is an adequate understanding of how the formation time is governed by the conditions in the gas out of which the star forms.

Our understanding of low-mass star formation is on a far better footing, since it has received much more observational and theoretical attention (Shu, Adams \& Lizano 1987). Low-mass stars form by accreting gas from a molecular "core" in which gravity overcomes thermal and nonthermal (magnetic and turbulent) pressure gradients. Shu (1977) considered the collapse of a singular isothermal sphere, finding

$$
\dot{m}_{*}=0.975 \frac{c_{\mathrm{th}}^{3}}{G}=4.36 \times 10^{-6}\left(\frac{T}{20 \mathrm{~K}}\right)^{3 / 2} M_{\odot} \mathrm{yr}^{-1}
$$

where $c_{\mathrm{th}}$ is the isothermal sound speed and the numerical evaluation assumes $n_{\mathrm{He}}=0.2 n_{\mathrm{H}_{2}}$. Observed temperatures of 10 to $20 \mathrm{~K}$ in regions of low-mass star formation imply accretion rates of about $10^{-6}$ to $10^{-5} M_{\odot} y^{-1}$, consistent with the inferred values of $t_{* f}$ for low-mass stars in these regions (Lada 1999).

There are two difficulties in extending this theory to high-mass stars. The first, discussed in some detail by Stahler et al. (2000), is that the predicted accretion rate depends only on the temperature of the gas. Once massive stars form, 
the gas may be heated to temperatures $\sim 50$ to $100 \mathrm{~K}$, but the first massive stars that form in a region will emerge from gas at $10-20 \mathrm{~K}$ and will have low accretion rates and correspondingly long formation times. The second difficulty is feedback from the massive stars. Since the Kelvin-Helmholtz contraction time is less than the accretion time for massive stars, they evolve along the main sequence while accreting. Massive protostars are thus very luminous, and it has been suggested that the radiation pressure and ionization they produce can halt the accretion and determine the upper limit of the stellar mass function (Larson \& Starrfield 1971; Kahn 1974; Wolfire \& Cassinelli 1987; Jijina \& Adams 1996). This feedback is so strong that it is impossible to form stars as massive as those observed if the accretion is assumed to be spherical, and the discrepancy grows as the accretion rate is reduced. These considerations have motivated the radical suggestion that massive stars form via the coalescence of low-mass stars in order to achieve a more rapid build up of the final stellar mass (Bonnell, Bate \& Zinnecker 1998).

Recently, McKee \& Tan (2002; hereafter MT) addressed the accretion-rate problem. The first step in resolving the problem of the apparently low accretion rates is to realize that, although equation (1) was derived for an isothermal gas, it should hold approximately when nonthermal support due to magnetic fields and turbulence is included as well (Stahler, Shu \& Taam 1980; Shu et al. 1987). Observed cores have turbulent motions that increase systematically with radius (Larson 1981; Caselli \& Myers 1995), and this leads to an increase in the accretion rate with time (Myers \& Fuller 1992; Caselli \& Myers 1995; MP97). Larger signal speeds allow for the hydrostatic support of denser gas cores, which then have shorter free-fall times and thus greater accretion rates once they become unstable. We term this the turbulent core model for massive star formation.

The second step in resolving the accretion rate problem is the recognition that massive stars form in regions of very high pressure and density. MT showed that for typical pressures in regions of massive star formation (Plume et al. 1997), stars form in a time of order $10^{5} \mathrm{yr}$. This result for the time scale is somewhat longer than that of Osorio et al. (1999), who inferred stellar masses and accretion rates by comparing calculated spectra with observations.

The purpose of this paper is several fold. First, we determine the relation between the pressure in a molecular cloud and its surface density $\Sigma$. We show that observed regions of massive star formation, both Galactic and extragalactic, have $\Sigma \sim 1 \mathrm{~g} \mathrm{~cm}^{-2}$, corresponding to mean pressures $\bar{P} / k \sim 4 \times 10^{8} \mathrm{~K} \mathrm{~cm}^{-3}$. Second, we extend the self-similar theory presented by MT to allow for magnetic fields and for a thermally supported core. Finally, we determine the radius and luminosity of accreting protostars, and use observed hot core luminosities to predict accretion rates and masses of several nearby massive protostars.

\section{THE PRESSURE AND SURFACE DENSITY OF REGIONS OF HIGH-MASS STAR FORMATION}

A cloud in hydrostatic equilibrium has a total pressure that is proportional to the square of the surface density, $P \sim G \Sigma^{2}$, where $\Sigma \equiv M(R) / \pi R^{2}$ (e.g., Elmegreen 1989). The Appendix contains a detailed discussion of this relation, and for our fiducial case - including the effects of magnetic fields and allowing for embedded stars - we find that the mean pressure in a cloud is typically $\bar{P} \simeq 0.88 G \Sigma^{2}$. What is the value of the surface density in regions of current and past massive star formation?

Regions of high-mass star formation studied by Plume et al. (1997) are characterized by virial masses $M_{\text {vir }} \sim 3800 M_{\odot}$ and radii $R \sim 0.5 \mathrm{pc}$. As discussed in the Appendix, the virial mass $M_{\text {vir }}$ is related to the actual mass $M$ by the virial parameter, $\alpha_{\mathrm{vir}}=M_{\mathrm{vir}} / M$. The virial parameter is about $1.3-1.4$ for GMCs, whereas it is quite close to unity for cores that are actively forming stars. Although there is no direct determination of $\alpha_{\text {vir }}$ for massive-star forming clumps, Plume et al. (1997) regard the virial mass as the most accurate estimate for the mass $\left(\alpha_{\mathrm{vir}} \simeq 1\right)$. Since this is consistent with the results for low-mass star formation discussed in the Appendix, we shall adopt $\alpha_{\text {vir }}=1$ for our numerical estimates. The mean column density in the clumps studied by Plume et al. is then $\Sigma_{\mathrm{cl}} \simeq 1 \mathrm{~g} \mathrm{~cm}^{-2}=4800 M_{\odot}$ pc ${ }^{-2}$; the corresponding visual extinction is $A_{V}=\left(N_{\mathrm{H}} / 2 \times 10^{21} \mathrm{~cm}^{-2}\right) \mathrm{mag}=214 \Sigma_{\mathrm{cl}}$ mag. From maps of a larger number of sources, Shirley et al (2002) find a median surface density (which they regard as a better characterization of their sample) from the virial theorem of $\Sigma_{\mathrm{cl}}=0.60 \mathrm{~g} \mathrm{~cm}^{-2}$. Mueller et al. (2002a,b) have determined the gas masses of a number of these sources from observations of the $350 \mu \mathrm{m}$ dust emission. Using the Ossenkopf and Henning (1994) opacities, they find gas masses that are on average 3.4 times lower than the virial mass. The mean surface density $\Sigma_{\mathrm{cl}}=0.19 \pm 0.12 \mathrm{~g} \mathrm{~cm}-2$ for the sources they were able to model in detail, while their total sample gives a higher value, $\Sigma_{\mathrm{cl}}=0.73 \pm 1.7 \mathrm{~g} \mathrm{~cm}^{-2}$. Had they used the Pollack et al. (1994) opacities instead, the inferred masses and surface densities would have been larger by a factor of about 2.5. These data show that there is a considerable dispersion in the mean surface density of the sources. These column densities are far greater than those associated with GMCs $\left(0.035 \mathrm{~g} \mathrm{~cm}^{-2}\right.$ _Solomon et al. 1987) or regions of low mass star formation (the average column density in the $\mathrm{C}^{18} \mathrm{O}$ cores in Taurus is $0.032 \mathrm{~g} \mathrm{~cm}^{-2}$ - Onishi et al. 1996).

We now compare the column density observed in regions of massive star formation with the surface density of stars in both Galactic and extragalactic star clusters. Before doing so, we note that there are several effects that can alter the relation between the observed stellar surface density and that of the molecular gas out of which the cluster formed. First, since not all the gas in the cloud goes into stars, some of the gas is ejected, causing the cluster to expand. For example, if the star formation were $50 \%$ efficient and the gas were lost slowly, then the cluster would expand by approximately a factor of two from its initial size (Hills 1980). Including the mass lost from the cluster implies that the initial surface density of gas would have been 8 times greater than the final surface density of stars. On the other hand, if magnetic fields were important in the support of the cloud, then the stellar velocity dispersion would have been less than the virial velocity (Patel \& Pudritz 1994), which would lead to an increase in the stellar surface density. If the size of the cluster is estimated from observations of the massive stars, then the resulting surface density will be larger than that of the total 
star cluster insofar as the massive stars form preferentially in the inner regions of clusters (Bonnell \& Davies 1998). This effect would be amplified if the cluster is old enough to have experienced significant mass segregation. Overall, we can expect the stellar surface density to be at best within a factor of a few of the initial gas surface density.

First, consider Galactic star clusters. The virial mass of the Orion Nebula Cluster (ONC), including both stars and gas, is about $4650 M_{\odot}$ (averaging the two models of Hillenbrand \& Hartmann 1998). About half the mass of the cluster is within $0.8 \mathrm{pc}$, giving $\Sigma \simeq 0.24 \mathrm{~g} \mathrm{~cm}^{-2}$. The Arches is a large cluster near the Galactic Center that contains many massive stars (Figer et al. 1999). Kim et al. (2000) have analyzed this cluster, taking into account the strong tidal effects experienced by a cluster so near the Galactic Center. They conclude that the initial mass of the cluster was about $2 \times 10^{4} M_{\odot}$. From their results, we estimate that the half-mass radius is about $0.4 \mathrm{pc}$, giving $\Sigma \simeq 4 \mathrm{~g} \mathrm{~cm}^{-2}$. Figer et al. infer a very flat IMF for the Arches $\left(d \mathcal{N}_{*} / d \ln m_{*} \propto m_{*}^{-0.6}\right.$ vs. $m_{*}^{-1.35}$ for the Salpeter IMF $)$. If the actual IMF of the Arches is closer to the Salpeter value and the heavy extinction has led to an underestimate of the number of low-mass stars, then the surface density would be larger than this estimate.

Globular clusters in the Galaxy no longer contain any massive stars, but they presumably did in their youth. There is a considerable dispersion in the properties of globular clusters; here we estimate the surface density of a typical one. The typical mass of a Galactic globular is about $2 \times 10^{5} M_{\odot}$ (inferred from Binney \& Merrifield 1998). The data summarized by van den Bergh, Morbey, \& Pazder (1991) give a median half-light radius for Galactic globulars of $R_{\mathrm{proj}, 1 / 2}=2.6 \mathrm{pc}$, where the subscript "proj" emphasizes that this is a projected radius. If the light traces the mass, then the spherical half-mass radius is $R_{1 / 2} \simeq 1.31 R_{\text {proj, } 1 / 2}$ (Spitzer 1987), which is 3.4 pc. The surface density inside the spherical half-mass radius is then $0.5 \mathrm{M} /\left(\pi R_{1 / 2}^{2}\right) \simeq 0.6 \mathrm{~g} \mathrm{~cm}^{-3}$. The corresponding value of the pressure, $\sim 3 \times 10^{8} \mathrm{~K} \mathrm{~cm}^{-3}$, is similar to the estimates of Elmegreen \& Efremov (1997) based on the same line of reasoning.

It has been suggested that super-star clusters (SSCs) in external galaxies are globular clusters in the process of formation (e.g., Ho \& Filippenko 1996). The SSCs NGC 1569-A1 and A2 each have masses of about $4 \times 10^{5} M_{\odot}$ (Gilbert \& Graham 2001 ) and projected half-light radii of about $1.7 \mathrm{pc}$ (DeMarchi et al 1997), corresponding to $\Sigma \simeq 2.7 \mathrm{~g} \mathrm{~cm}^{-2}$. A particularly dramatic SSC is the one observed by Turner, Beck, \& Ho (2000) in NGC 5253: they estimate that a star cluster with total ionizing luminosity of $\sim 4 \times 10^{52} \mathrm{~s}^{-1}$ is confined within a region about $1 \times 2 \mathrm{pc}$ in diameter. For zero age main sequence models (Schaerer \& de Koter 1997), this corresponds to a total stellar mass of $0.6-1.5 \times 10^{6} M_{\odot}$, for Salpeter extrapolation to 1 and $0.1 M_{\odot}$, respectively. If we take the projected half-mass radius to be $0.75 \mathrm{pc}$, then $\Sigma \simeq 20-50 \mathrm{~g}$ $\mathrm{cm}^{-2}$.

These results are collected in Table 1. It is striking that both the molecular regions where massive stars are forming and the star clusters that formed from them all have surface densities $\Sigma \sim 1 \mathrm{~g} \mathrm{~cm}^{-2}$ (with the exception of the cluster in NGC 5253, which is somewhat larger). We first characterize the properties of molecular gas in such an environment and then determine the time scale for star formation.

\section{SELF-SIMILAR CORES AND CLUMPS}

Molecular clouds are inhomogeneous on a wide range of scales (Williams, Blitz, \& McKee 2000), and numerical simulations show that this is a natural outcome of supersonic turbulence, both with and without magnetic fields (VazquezSemadeni et al. 2000). Following Williams et al., we define a star-forming clump as a massive region of molecular gas out of which a star cluster is forming; a core is a region of molecular gas that will form a single star (or multiple star system

TABLE 1

Characteristic Surface Densities of Regions of High-Mass Star Formation

\begin{tabular}{ccccc}
\hline \hline Object [Ref.] & $M\left(M_{\odot}\right)$ & $R_{1 / 2}(\mathrm{pc})$ & $\Sigma\left(\mathrm{g} \mathrm{cm}^{-2}\right)$ & $\bar{P}_{\mathrm{cl}} / k\left(\mathrm{~K} \mathrm{~cm}{ }^{-3}\right)$ \\
\hline Galactic Star-forming Clumps [1] & $3800^{\mathrm{b}, c}$ & $0.5^{\mathrm{c}}$ & 1.0 & $4 \times 10^{8}$ \\
Orion Nebula Cluster [2] & $4600^{\mathrm{b}}$ & 0.8 & 0.24 & $2 \times 10^{7}$ \\
Arches Cluster [3,4] & $2 \times 10^{4}$ & 0.4 & 4 & $7 \times 10^{9}$ \\
Galactic Globular Clusters [5,6] & $2 \times 10^{5 \mathrm{~b}}$ & 3.4 & 0.8 & $3 \times 10^{8}$ \\
NGC1569-A1,A2 [7,8] & $4 \times 10^{5 \mathrm{~b}}$ & 2.2 & 2.7 & $3 \times 10^{9}$ \\
NGC5253 [9] & $0.6-1.5 \times 10^{6 \mathrm{~d}}$ & 1.0 & $20-50$ & $2-11 \times 10^{11}$ \\
\hline
\end{tabular}

a References: (1) Plume et al. (1997); (2) Hillenbrand \& Hartmann (1998); (3) Figer et al. (1999); (4) Kim et al. (2000); (5) Binney \& Merrifield (1998); (6) van den Bergh et al. (1991); (7) Gilbert \& Graham (2001); (8) DeMarchi et al. (1997); (9) Turner, Beck, \& Ho (2000)

\footnotetext{
${ }^{\mathrm{b}}$ Virial mass estimates

${ }^{{ }^{c}}$ The half-mass radius is not well-defined for the Plume et al. (1997) clouds, since the mass distribution on larger scales is not known. We therefore evaluate $\Sigma=M / \pi R^{2}$ using the typical radius and virial mass that they observe.

${ }^{\mathrm{d}}$ Extrapolation from inferred LyC luminosity of H II region based on Salpeter IMF with a lower mass limit $m_{\ell}=$ 1, $0.1 M_{\odot}$.
} 
such as a binary). Star-forming clumps are observed to be approximately gravitationally bound (Bertoldi \& McKee 1992), whereas cores are necessarily gravitationally bound.

Our basic assumption is that a star-forming clump and the cores embedded within it are each part of a self-similar, self-gravitating turbulent structure on all scales above that of the thermal Jeans mass (MT), an assumption that appears to be in accord with observation (Williams et al. 2000). Except on small scales, the turbulence is therefore necessarily supersonic, and we shall show that this is self-consistent below. We further assume that the structure is approximately stationary in time (i.e., it is not in a state of dynamical collapse or expansion); it follows that the star-forming clump and most of the cores within it are in approximate hydrostatic equilibrium. However, some of the cores are gravitationally unstable and are collapsing on a dynamical time scale - these are the cores that are in the process of forming stars. Finally, we assume that the clump and the cores within it are approximately spherical. As shown by Bertoldi \& McKee (1992), deviations from sphericity of a factor of a few are readily accounted for; in any case, observations of high-mass star forming clumps show that they are approximately spherical (Shirley et al. 2002).

This model for regions of massive star formation is necessarily highly approximate. Regions of massive star formation are observed to have highly supersonic velocity dispersions (Plume et al. 1997) and are presumably turbulent. Large amplitude density fluctuations in such regions continually form, grow, and dissipate. Since we have assumed that a clump has a lifetime that is at least several times greater than its free-fall time, it follows that only a small fraction of the mass of the clump can be contained in density fluctuations that are gravitationally bound and are undergoing gravitational collapse - i.e., in cores that are in the process of star formation. The same conclusion applies to massive cores, which are themselves turbulent; if on the contrary, most of the mass of a core were in density fluctuations that were undergoing gravitational collapse, then the core would form a cluster of low-mass stars instead of a massive star. Calculations of turbulent, self-gravitating clouds indicate that indeed only a small fraction of the mass is gravitationally unstable at any given time (e.g., Vazquez-Semadeni, Ballesteros-Paredes, \& Klessen 2002).

In this section we shall work out the properties of this self-similar structure, leaving consideration of the effects of thermal pressure to $\S 5$. The results in this section apply equally well to an individual core or to a clump. For clumps, we allow for the possibility that only a fraction $f_{\mathrm{g}}$ of the mass of the clump is gaseous, with the rest in stars. If stars are present, we assume that their spatial distribution is identical to that of the gas. We do not attempt to evaluate the mass distribution of the cores, which is related to the initial mass function (IMF) of the stars that form; instead, this mass distribution is assumed to be such that it leads to the observed IMF. Presumably this mass distribution is determined by the characteristics of the turbulence in the star-forming clump (e.g., Henriksen \& Turner 1984; Elmegreen \& Falgarone 1996).

In a self-similar, spherical medium, the density and pressure each have a power-law dependence on radius, $\rho \propto r^{-k_{\rho}}$ and $P \propto r^{-k_{P}}$. It follows that the sphere is a polytrope with $P \propto \rho^{\gamma_{p}}$. In hydrostatic equilibrium we have (MP96; McKee \& Holliman 1999)

$$
k_{\rho}=\frac{2}{2-\gamma_{p}}, \quad k_{P}=\gamma_{p} k_{\rho}=\frac{2 \gamma_{p}}{2-\gamma_{p}}=2\left(k_{\rho}-1\right) .
$$

Let $c \equiv(P / \rho)^{1 / 2}$ be the effective sound speed. The equation of hydrostatic equilibrium then gives

$$
M=\frac{k_{P} c^{2} r}{G}
$$

where $M$ is the total mass of the system, including any stars that are present. It follows that the gas density is

$$
\rho=\frac{A c^{2}}{2 \pi G r^{2}}
$$

where

$$
A=\left(3-k_{\rho}\right)\left(k_{\rho}-1\right) f_{\mathrm{g}}=\frac{\gamma_{p}\left(4-3 \gamma_{p}\right) f_{\mathrm{g}}}{\left(2-\gamma_{p}\right)^{2}}
$$

(cf. MP96).

It is convenient to express the properties of the cores and clumps in terms of the pressure and mass. Since $\rho=P / c^{2}$, equations (3) and (4) give

$$
\begin{gathered}
c=\left(\frac{2 \pi G^{3} M^{2} P}{A k_{P}^{2}}\right)^{1 / 8}, \\
r=\left(\frac{A G M^{2}}{2 \pi k_{P}^{2} P}\right)^{1 / 4}
\end{gathered}
$$

and

$$
\rho=\left(\frac{A k_{P}^{2} P^{3}}{2 \pi G^{3} M^{2}}\right)^{1 / 4} .
$$

In this subsection, $A$ can refer either to clumps, which generally have $f_{\mathrm{g}}<1$, or to cores, which are assumed to have $f_{\mathrm{g}}=1$; in the remainder of the paper, however, we shall use $A$ to refer only to cores. 
Observe that the only dependence of these properties on the polytropic index $\gamma_{p}$, or equivalently, on the power-law indices $k_{P}$ and $k_{\rho}$, is in the factors $A$ and $k_{P}$, which are raised to relatively low powers. Studies of low-mass star formation often adopt the singular isothermal sphere as a model (Shu 1977), which appears to be approximately consistent with observation (Andre, Ward-Thompson, \& Barsony 2000). Such a model has $\gamma_{p}=1$, so that $k_{\rho}=k_{P}=2$ and $A=1$. No data are available on the structure of cores that are forming very massive stars. MP96 and MP97 have discussed an alternative model, the logatrope, in which the pressure varies as the logarithm of the density, but as discussed in Appendix $\mathrm{B}$, this model is not physically realistic. Observations of molecular clouds show that the velocity dispersion $\sigma$ increases outwards (Larson 1981). Since we expect that $\sigma \propto c$, and since $c^{2}=P / \rho \propto r^{2-k_{\rho}}$, this implies that $k_{\rho}<2$ and $\gamma_{p}<1$ (Maloney 1988). (If $\gamma_{p}$ is written as $\gamma_{p}=1+1 / n$, this corresponds to a negative index $n$; such a polytrope is therefore referred to as a "negative index polytrope.")

For "high-mass" cores in Orion, Caselli \& Myers (1995) find $k_{\rho} \simeq 1.45$ with a dispersion of \pm 0.2 . According to van der Tak et al. (2000), the clumps in which high-mass cores are embedded have values of $k_{\rho}$ ranging from 1 to 2 , centered around 1.5. ${ }^{1}$ Beuther et al. (2002) find $k_{\rho} \simeq 1.6 \pm 0.5$ for their sample of massive star-forming regions, while Mueller et al. (2002b) find $k_{\rho} \simeq 1.8 \pm 0.4$ for a sample of 31 sources (see Evans et al. 2002 for a review). For our numerical estimates of the properties of both high-mass cores and clumps, we shall adopt $k_{\rho}=1.5$, which corresponds to $\gamma_{p}=2 / 3, k_{P}=1$, and $A=3 / 4$. For comparison, the value $k_{\rho}=1.8$ found by Mueller et al. (2002b) corresponds to $\gamma_{p}=8 / 9, k_{P}=1.6$, and $A=0.96$.

The value of $\gamma_{p}$ determines the structure of a polytrope. As discussed by McKee \& Holliman (1999), the stability of the polytrope depends on the adiabatic index $\gamma$ as well as on $\gamma_{p}$. If the polytrope is isentropic $\left(\gamma=\gamma_{p}\right)$, then the maximum density contrast between the center and edge of a stable molecular cloud is quite limited $\left(\leq 14\right.$ for $\left.\gamma_{p} \leq 1\right)$. On the other hand, for non-isentropic clouds, an infinite density contrast is possible in a stable cloud provided $\gamma$ exceeds a critical value; for our fiducial case of $\gamma_{p}=2 / 3$, this critical value of $\gamma$ is unity. The value of $\gamma$ for turbulent magnetic fields is $4 / 3$, significantly above this critical value. The effective value of $\gamma$ for the turbulent motions in molecular clouds is not known; this is a thorny problem, since the motions are inferred to damp very rapidly (Vazquez-Semadeni et al. 2000). The large density contrasts observed in molecular clouds suggest that, insofar as polytropic models are applicable, the effective value of $\gamma$ is large enough to render the clouds stable against dynamical collapse.

\subsection{The Mean Pressure in Clumps}

The mean pressure in a clump is $\bar{P}_{\mathrm{cl}} \equiv\left(1 / V_{\mathrm{cl}}\right) \int P d V$. As discussed in Appendix A, this pressure is proportional to $G \Sigma_{\mathrm{cl}}^{2}$, so we write

$$
\bar{P}_{\mathrm{cl}} \equiv \phi_{\bar{P}} G \Sigma_{\mathrm{cl}}^{2}
$$

where $\phi_{\bar{P}}$ is a numerical factor of order unity. From equation (A5), we find

$$
\phi_{\bar{P}}=\left(\frac{3 \pi}{20}\right) f_{\mathrm{g}} \phi_{\mathrm{geom}} \phi_{B} \alpha_{\mathrm{vir}},
$$

where $\phi_{\text {geom }}$ represents the effect of non-spherical geometry and $\phi_{B} \equiv\left\langle c^{2}\right\rangle /\left\langle\sigma^{2}\right\rangle$ the effect of magnetic fields. Here $\sigma$ is the one dimensional velocity dispersion; in the absence of magnetic fields, this is identical to the effective sound speed $c$, and $\phi_{B}=1$. Insofar as the clump can be approximated as part of a singular polytropic sphere, equations (A8) and (A9) show that the product $\phi_{B} \alpha_{\text {vir }}$ is well determined.

As discussed in the Appendix, clouds with aspect ratios of 2:1, either oblate or prolate, have $\phi_{\text {geom }} \simeq 1.1$. Observed low-mass cores have aspect ratios of about 3:1 if they are oblate and 2:1 if they are prolate (Myers et al. 1991). Basu (2000) considered triaxial clouds and argued against larger aspect ratios for oblate clouds, which are formally allowed by the observations. Although the shape of high-mass cores is unknown, high-mass clumps appear to have quite spherical shapes (Shirley et al. 2002); we therefore adopt $\phi_{\text {geom }}=1$ for our fiducial case. Observations suggest that the Alfven Mach number is of order unity, and in that case the discussion in the Appendix shows that $\phi_{B} \simeq 2.8$. (By contrast, MT considered the non-magnetic case with $\phi_{B}=1$.) The factor $f_{\mathrm{g}}$ measures the fraction of the cloud mass in gas. Although the star formation efficiency averaged over all the molecular gas in the Galaxy is relatively small $(\sim 5 \%$ over the life of an association according to Williams \& McKee 1997), we anticipate that the efficiency in the dense clumps forming massive stars could be considerably higher. We assume that a typical massive star-forming clump has about $1 / 3$ of its mass in stars, corresponding to $f_{\mathrm{g}}=2 / 3$. Finally, as discussed in the Appendix, the virial parameter $\alpha_{\mathrm{vir}}$ is about unity in star-forming regions. Adopting these fiducial values for the parameters that enter $\phi_{\bar{P}}$, we find that the fiducial value is then $\phi_{\bar{P}} \simeq 0.88$, so that $\bar{P}_{\mathrm{cl}}=0.88 G \Sigma_{\mathrm{cl}}^{2}$ and

$$
\bar{P}_{\mathrm{cl}} / k=4.25 \times 10^{8} \Sigma_{\mathrm{cl}}^{2} \quad \mathrm{~K} \mathrm{~cm}^{-3} .
$$

1 van der Tak et al. (2000) remark that $k_{\rho}=1.5$ is just what is expected for regions undergoing supersonic infall. In our model, this region should not extend beyond the core radius - i.e., the radius of the region that is collapsing to form a massive star. The core radius has a fiducial value $R_{\text {core }} \sim 0.06$ pc (see eq. 19), which corresponds to an angle of only a few arcsec at the typical distance of several kpc for observed massive star-forming regions. The beam sizes used to observe these regions are generally larger than a few arcsec, so the observed values of $k_{\rho}$ should typically refer to the clump in which the core is embedded rather than to the core itself. A central assumption of our model is that these star-forming clumps are in approximate hydrostatic equilibrium, which implies that the observed values of $k_{\rho}$ reflect the structure of this equilibrium, not a dynamical collapse. 


\subsection{Properties of Cores}

We are now in a position to express the properties of a core out of which a massive star forms in terms of the pressure of the clump in which it is embedded and the mass of the star that will form. The key point is that, on average, the pressure at the surface of a core will be the same as the ambient pressure in the clump there, $P_{s, \text { core }}=P_{\mathrm{cl}}(r)$. We define the parameter $\phi_{P \text {, core }}$ as the ratio of this pressure to the mean pressure in the clump. Since the clump is self-similar by assumption, we have

$$
\phi_{P, \text { core }} \equiv \frac{P_{\mathrm{cl}}(r)}{\bar{P}_{\mathrm{cl}}}=\frac{3-k_{P}}{3}\left(\frac{r}{R_{\mathrm{cl}}}\right)^{-k_{P}}=\frac{5-2 k_{\rho}}{3}\left[\frac{M(r)}{M_{\mathrm{cl}}}\right]^{-2\left(k_{\rho}-1\right) /\left(3-k_{\rho}\right)} .
$$

For example, if the clump is a singular isothermal sphere $\left(k_{P}=k_{\rho}=2\right)$, then $\phi_{P \text {, core }}=\frac{1}{3}\left[M(r) / M_{\mathrm{cl}}\right]^{-2}$; for our fiducial case of $k_{\rho}=1.5$, we have $\phi_{P, \text { core }}=\frac{2}{3}\left[M(r) / M_{\mathrm{cl}}\right]^{-(2 / 3)}$.

What is the typical value of $\phi_{P \text {, core }}$ ? If the cores trace the mass of the clump, then for $k_{\rho}=1.5$ the value of $\phi_{P \text {, core }}$ at the half-mass point $\left(M=0.5 M_{\mathrm{cl}}\right)$ is 1.06. However, Bonnell and Davies (1998) have argued that, at least in the case of the Orion Nebula Cluster (ONC), the massive stars formed preferentially in the inner parts of the cluster. In particular, the Trapezium stars originated within about $30 \%$ of the half-mass radius of the ONC. If we assume that the ONC is similar to the Plume et al. clumps and adopt $0.3 R_{1 / 2}$ as the typical radius of formation of massive stars, then

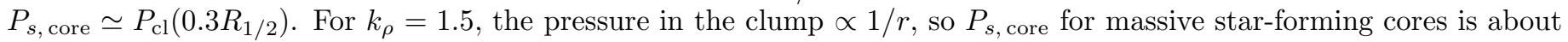
3 times that at the clump surface (recall that we are identifying $R_{1 / 2}$ with the radius of the Plume et al. clumps), which in turn is a factor $\left(3-k_{P}\right) / 3=2 / 3$ times the mean pressure inside the clump; hence, $\phi_{P, \text { core }} \simeq 2$ in this case. We shall adopt this as a fiducial value.

The pressure at the surface of the core is then related to the surface density of the clump by

$$
P_{s, \text { core }}=\phi_{P, \text { core }} \phi_{\bar{P}} G \Sigma_{\mathrm{cl}}^{2} \text {. }
$$

Numerically, we have

$$
P_{s, \text { core }} / k=4.83 \times 10^{8} \phi_{P, \text { core }} \phi_{\bar{P}} \Sigma_{\mathrm{cl}}^{2} \quad \mathrm{~K} \mathrm{~cm}^{-3} \rightarrow 0.85 \times 10^{9} \Sigma_{\mathrm{cl}}^{2} \quad \mathrm{~K} \mathrm{~cm}^{-3},
$$

where the final expression is for the fiducial case $\left(\phi_{P, \text { core }}=2.0\right.$ and $\left.\phi_{\bar{P}}=0.88\right)$.

To express the core properties in terms of the final mass of the star that will form out of it, $m_{* f}$, we note that only a fraction $\epsilon_{\text {core }}$ of the mass of the core will end up in the star; the rest is dispersed by the powerful protostellar outflow associated with the star as it forms (e.g., Matzner \& McKee 2000). We assume that the star formation efficiency $\epsilon_{\text {core }}$ is constant throughout the accretion process, so that at any time the mass of a star, $m_{*}$, is related to the mass of gas out of which it formed, $M_{\text {core }}$, by

$$
m_{*}=\epsilon_{\mathrm{core}} M_{\text {core }} .
$$

The value of $\epsilon_{\text {core }}$ is uncertain for massive stars, since it depends on the unknown properties of protostellar winds from massive stars. For low-mass stars, $\epsilon_{\text {core }} \sim 0.25-0.75$ (Matzner \& McKee 2000). A similar analysis involving the scaling of low-mass outflows to high-mass protostars stars yields similar efficiencies (Tan \& McKee 2002a, Paper II), so for numerical estimates we shall adopt $\epsilon_{\mathrm{core}}=0.5$ as a typical value.

We begin with the one-dimensional velocity dispersion $\sigma=c / \phi_{B}^{1 / 2}$. Since $\phi_{B}$ is a number of order unity, $\sigma$ and $c$ scale in the same way with $r$ :

$$
\sigma \propto c \propto r^{\left(2-k_{\rho}\right) / 2} \propto M^{\left(2-k_{\rho}\right) / 3\left(3-k_{\rho}\right)} .
$$

Equation (6) implies that the velocity dispersion at the surface of a core is given by

$$
\begin{aligned}
\sigma_{s}=\frac{c_{s}}{\phi_{B}^{1 / 2}} & =1.61\left(\frac{\phi_{P, \text { core }} \phi_{\bar{P}}}{A k_{P}^{2} \phi_{B}^{4} \epsilon_{\mathrm{core}}^{2}}\right)^{1 / 8}\left(\frac{m_{* f}}{30 M_{\odot}}\right)^{1 / 4} \Sigma_{\mathrm{cl}}^{1 / 4} \mathrm{~km} \mathrm{~s}^{-1}, \\
& \rightarrow 1.27\left(\frac{m_{* f}}{30 M_{\odot}}\right)^{1 / 4} \Sigma_{\mathrm{cl}}^{1 / 4} \mathrm{~km} \mathrm{~s}^{-1}
\end{aligned}
$$

where the last value is for the fiducial case. This velocity dispersion is considerably greater than the isothermal sound speed, $c_{\text {th }}=0.27(T / 20 \mathrm{~K})^{1 / 2} \mathrm{~km} \mathrm{~s}^{-1}$. As a result, the cores that form massive stars are necessarily supersonically turbulent and therefore clumpy. This is one of the principal results of this paper. It is often assumed that star formation can occur only when the gas becomes subsonic (e.g., Henriksen 1986; Vazquez-Semadeni et al. 2002), and this assumption leads directly to the problems with massive star formation that have been cogently summarized by Stahler et al. (2000). In our view, this assumption is unjustified, and we shall show that we can obtain a theory of massive star formation that appears to be consistent with the data by dropping it.

The radius of a core is (eq. 7)

$$
\begin{aligned}
R_{\text {core }} & =0.050\left(\frac{A}{k_{P}^{2} \epsilon_{\text {core }}^{2} \phi_{P, \text { core } \phi_{\bar{P}}}}\right)^{1 / 4}\left(\frac{m_{* f}}{30 M_{\odot}}\right)^{1 / 2} \Sigma_{\mathrm{cl}}^{-1 / 2} \mathrm{pc} \\
& \rightarrow 0.057\left(\frac{m_{* f}}{30 M_{\odot}}\right)^{1 / 2} \Sigma_{\mathrm{cl}}^{-1 / 2} \mathrm{pc} .
\end{aligned}
$$


Finally, the density of $\mathrm{H}$ atoms at the surface of a core is (eq. 8)

$$
\begin{aligned}
n_{\mathrm{H}, s} & =1.10 \times 10^{6}\left(A k_{P}^{2} \epsilon_{\text {core }}^{2} \phi_{P, \text { core }}^{3} \phi_{\bar{P}}^{3}\right)^{1 / 4}\left(\frac{m_{* f}}{30 M_{\odot}}\right)^{-1 / 2} \Sigma_{\mathrm{cl}}^{3 / 2} \mathrm{~cm}^{-3} \\
& \rightarrow 1.11 \times 10^{6}\left(\frac{m_{* f}}{30 M_{\odot}}\right)^{-1 / 2} \Sigma_{\mathrm{cl}}^{3 / 2} \mathrm{~cm}^{-3} .
\end{aligned}
$$

Note that equations for the clump velocity dispersion, radius, and density can be obtained from equations (17), (19), and (21) by making the replacements $m_{* f} / \epsilon_{\text {core }} \rightarrow M_{\mathrm{cl}}$ and $A \rightarrow A_{\text {core }} f_{\mathrm{g}}$; by setting $\phi_{P \text {, core }}=\left(3-k_{P}\right) / 3$ so as to set the pressure equal to its value at the surface of the clump (eq. 12); and by setting $\alpha_{\mathrm{vir}} \phi_{B}=\alpha_{\mathrm{SPS}}$ so as to ensure that the clump is in hydrostatic equilibrium (eq. A8).

How are the core properties related to those of the clump in which it is embedded? Consider first the surface density of a core, $\Sigma_{\text {core }} \equiv\left(m_{* f} / \epsilon_{\text {core }}\right) / \pi R_{\text {core }}^{2}$ Equations (7) and (13) give

$$
\frac{\Sigma_{\text {core }}}{\Sigma_{\mathrm{cl}}}=\left(\frac{2 k_{P}^{2}}{A \pi} \phi_{P, \text { core }} \phi_{\bar{P}}\right)^{1 / 2} \rightarrow 1.22 \text {. }
$$

The cores thus have a column density comparable to that of the clump as a whole. Note that $\Sigma_{\mathrm{cl}}$ includes the mass of the stars in the clump; the ratio of $\Sigma_{\text {core }}$ to the column density of gas in the clump is larger by a factor $1 / f_{\mathrm{g}}$.

One of the important questions that must be addressed in any model for the formation of dense star clusters is how a large number of protostellar cores can fit into the small volume of the clump in which the stars are forming. This issue is automatically solved in our model since the cores are much denser than the clumps. We compare the mean density in a core, $\bar{\rho}_{\text {core }}$, to the mean gas density in the central part of a clump, $\rho_{\mathrm{cl}}=\phi_{\rho \text {, core }} \bar{\rho}_{\mathrm{cl}}=\phi_{\rho \text {, core }} 3 f_{\mathrm{g}} M /\left(4 \pi R^{3}\right)$, where $\bar{\rho}_{\mathrm{cl}}$ is the mean overall clump density and the factor $\phi_{\rho \text {, core }}=\left(3-k_{\rho}\right) / 3 \cdot\left(r / R_{\mathrm{cl}}\right)^{-k_{\rho}}$ allows for central concentration. As before we assume the typical high-mass star-forming core is located at $r=R_{\mathrm{cl}} / 3$ (Bonnell \& Davies 1998), so that for $k_{\rho}=1.5$, $\phi_{\rho, \text { core }} \simeq 2.6$. With the aid of equations (7) and (13) we find

$$
\begin{aligned}
\frac{\bar{\rho}_{\text {core }}}{\rho_{\mathrm{cl}}} & =\frac{1}{\phi_{\rho, \text { core }}}\left(\frac{3 \phi_{P, \text { core }}}{3-k_{P}}\right)^{3 / 4} \frac{1}{f_{\mathrm{g}}^{1 / 4}}\left(\frac{\epsilon_{\text {core }} M}{m_{* f}}\right)^{1 / 2} \\
& \rightarrow 7.9\left(\frac{M_{\mathrm{cl}}}{4000 M_{\odot}}\right)^{1 / 2}\left(\frac{30 M_{\odot}}{m_{* f}}\right)^{1 / 2} .
\end{aligned}
$$

The mean density of stellar mass cores is about an order of magnitude larger than the mean gas density in the surrounding clump. Thus, even though the clump is forming a very dense star cluster, there is no "crowding problem."

Finally, we compare the core radius with the tidal radius in the clump; for our model to be self-consistent, we require the core radius to be less than the tidal radius. If the clump were a rotationally supported disk, then the tidal radius is $R_{t} \simeq 0.46\left[M_{\text {core }} / M(r)\right]^{1 / 3} r$, where $r$ is the distance from the core to the center of the clump and $M(r)$ is the clump mass within $r$ (Paczynski 1971). For Galactic star-forming clumps, this is somewhat smaller than the core radius inferred from equation (7), $R_{\text {core }}=f_{\mathrm{g}}^{-1 / 4}\left[M_{\text {core }} / M(r)\right]^{1 / 2} r$. However, molecular cloud cores in low-mass star-forming regions show little evidence for rotation (Goodman et al. 1992), and we assume that this remains true in regions of high-mass star formation. In that case, the clump is supported by turbulent motions and magnetic fields, and the tidal radius is determined by

$$
\left(k_{\rho}-1\right)\left[\frac{G M(r)}{r^{3}}\right] R_{t}=\frac{G M_{\text {core }}}{R_{t}^{2}},
$$

where we have assumed $k_{\rho}>1$. The ratio of the tidal radius to the core radius,

$$
\frac{R_{t}}{R_{\text {core }}}=\frac{f_{\mathrm{g}}^{1 / 4}}{\left(k_{\rho}-1\right)^{1 / 3}}\left[\frac{M(r)}{M_{\text {core }}}\right]^{1 / 6},
$$

is then somewhat greater than unity, as required. Note that this ratio increases weakly with the clump mass, so that the omission of effects associated with tidal gravitational fields becomes a better approximation for more massive star-forming clumps.

\section{SELF-SIMILAR HIGH-MASS STAR FORMATION}

Having determined the properties of massive star-forming clumps and the cores embedded within them, we now consider the rate at which a protostar grows once the core from which it is forming becomes gravitationally unstable. Dimensional arguments indicate that the accretion rate should be $\dot{m}_{*} \sim M_{\text {core }} / t_{\mathrm{ff}}$, where $t_{\mathrm{ff}}=(3 \pi / 32 G \rho)^{1 / 2}$ is the free-fall time (Stahler, Shu, \& Taam 1980; see also Shu et al 1987). Following MT, we write this as

$$
\dot{m}_{*}=\phi_{*} \frac{m_{*}}{t_{\mathrm{ff}}}
$$

where $\phi_{*}$ is a dimensionless constant of order unity. This equation has the same dependence on dimensional parameters as equation (1) if $c_{\mathrm{th}}$ is replaced by the virial velocity $\left(G m_{*} / R\right)^{1 / 2}$ (Stahler et al 1980; Shu et al. 1987), since $\left(G m_{*} / R\right)^{3 / 2} / G \propto$ $m_{*}\left(G m_{*} / R^{3}\right)^{1 / 2} \propto m_{*} / t_{\mathrm{ff}}$. More precisely, equations (3), (4), and (28) imply

$$
\dot{m}_{*}=\phi_{*} \epsilon_{\text {core }} \frac{4}{\pi \sqrt{3}} k_{P} A^{1 / 2} \frac{c^{3}}{G}
$$


which is the generalization of equation (1) to the non-isothermal case.

Equation (28) could be violated in the sense that $\phi_{*}$ is much greater than unity only in the unlikely case that the star forms from a coherent spherical implosion; if the star formation is triggered by an external increase in pressure, $\phi_{*}$ could be increased somewhat, but deviations from spherical symmetry in the triggering impulse and in the protostellar core will generally prevent $\phi_{*}$ from becoming too large. The equation could be violated in the opposite sense that $\phi_{*}$ is much less than unity if the core is magnetically dominated, so that collapse could not begin until the magnetic field diffused out of the core. However, the magnetic energy in star-forming cores is generally less than or about equal to the gravitational energy (Crutcher 1999), so this effect is unlikely to be significant. Note that since the core out of which a massive star forms is highly turbulent, it will be clumpy and the accretion rate can be expected to show large fluctuations-i.e., in reality $\phi_{*}$ will be a random function of time.

MT showed that if the collapse is spherical and self-similar, then $\phi_{*}$ is quite close to unity provided that the value of $\rho$ entering $t_{\mathrm{ff}}$ is evaluated at the radius in the initial core that just encloses the gas that goes into the star when its mass is $m_{*}$. The resulting value of $\phi_{*}$ averages over the fluctuations that would occur in an actual turbulent core. For a polytropic sphere, $\rho \propto r^{-k_{\rho}}$ and $M \propto r^{3-k_{\rho}}$, which implies $\rho \propto M^{-k_{\rho} /\left(3-k_{\rho}\right)}=M^{2 /\left(4-3 \gamma_{p}\right)}$. Since we have assumed that the stellar mass is a constant fraction $\epsilon_{\text {core }}$ of the gas mass (see eq. 15), the accretion rate varies as

$$
\dot{m}_{*} \propto m_{*} \rho^{1 / 2} \propto m_{*}^{1-1 /\left(4-3 \gamma_{p}\right)} .
$$

Integration yields

$$
m_{*}=m_{* f}\left(\frac{t}{t_{* f}}\right)^{4-3 \gamma_{p}},
$$

where $m_{* f}$ is the final stellar mass, which is attained at a time $t_{* f}$ (MP97). This equation implies

$$
\dot{m}_{*}=\left(4-3 \gamma_{p}\right) \frac{m_{*}}{t}=\left(4-3 \gamma_{p}\right) \frac{m_{* f}}{t_{* f}}\left(\frac{t}{t_{* f}}\right)^{3-3 \gamma_{p}} .
$$

Note that for $\gamma_{p}<1$ the accretion rate accelerates (MP97); in particular, for the fiducial case $\gamma_{p}=2 / 3$, we have $\dot{m}_{*} \propto t$. From equations (28) and (32), the star-formation time, which is the time from the first formation of a protostellar core to the time the star reaches its final mass, is then

$$
t_{* f}=\frac{\left(4-3 \gamma_{p}\right)}{\phi_{*}} t_{\mathrm{ff}, s}
$$

where $t_{\mathrm{ff}, s}$ is the value of the free-fall time evaluated at the surface of the core-i.e., for the last parcel of gas to accrete onto the star.

As discussed by MP97, termination of the accretion breaks the self-similarity once the expansion wave reaches $m_{* f}$. This occurs at a time they label $t_{\mathrm{ew}}$, which is in the range $(0.42-0.49) t_{* f}$ for $0 \leq \gamma_{p} \leq 1$. If the boundary of the core is at constant pressure, a compression wave will be launched inward when the expansion wave reaches the boundary (Stahler et al. 1980). Equation (32) remains accurate until this compression wave reaches the center. Numerical calculations by Reid, Pudritz, \& Wadsley (2002) show that in the logatropic case (which is related to the polytropic case with $\gamma_{p} \rightarrow 0$ ) this occurs at a time of about $0.7 t_{* f}$. Thereafter, the accretion rate gradually decreases.

MT used the results of MP97 to evaluate $\phi_{*}$ in the non-magnetic case, which we label $\phi_{* \text { non }}$ :

$$
\begin{aligned}
\phi_{* \text { non }} & =\left(4-3 \gamma_{p}\right) \frac{t_{\mathrm{ff}, s}}{t_{* f}}, \\
& =\pi \sqrt{ } 3\left[\frac{\left(2-\gamma_{p}\right)^{2}\left(4-3 \gamma_{p}\right)^{\left(7-6 \gamma_{p}\right) / 2} m_{0}}{8^{\left(5-3 \gamma_{p}\right) / 2}}\right]^{1 /\left(4-3 \gamma_{p}\right)},
\end{aligned}
$$

(see Fig. 1), where $m_{0}\left(\gamma_{p}\right)$ is a parameter that is evaluated by MP97. ${ }^{2}$ For example, for the singular isothermal sphere, we have $m_{0}=0.975$ and $\phi_{* \text { non }}=0.975 \pi \sqrt{3} / 8=0.663$. For other values of $\gamma_{p}$ in the range $0 \leq \gamma_{p} \leq 1$, which is equivalent to $1 \leq k_{\rho} \leq 2$, the approximation

$$
\phi_{* \text { non }} \simeq 1.62-0.48 k_{\rho}
$$

is accurate to within about $1 \%(\mathrm{MT})$. For the fiducial case $\left(k_{\rho}=1.5\right)$, we have $\phi_{* \text { non }}=0.90$. We conclude that $\dot{m}_{*} \simeq m_{*} / t_{\mathrm{ff}}$ to within a factor 1.5 for non-magnetic, spherical cores in which the effective sound speed increases outward.

We can estimate the effect of magnetic fields on the accretion rate from the work of $\mathrm{Li} \& \mathrm{Shu}$ (1997), who considered collapse of self-similar, isothermal, magnetized, toroidal clouds. The equilibrium surface density is $\Sigma=\left(1+H_{0}\right) c_{\mathrm{th}}^{2} /(\pi G \varpi)$, where $\varpi$ is the cylindrical radius and $H_{0}$ is a parameter that increases from zero as the magnetic field is increased. They show that the accretion rate is $\dot{m}_{*}=1.05\left(1+H_{0}\right) c_{\mathrm{th}}^{3} / G$, which is larger than that in equation (1) by about a factor $\left(1+H_{0}\right)$. However, equation (28) predicts $\dot{m}_{*} \propto M \rho^{1 / 2} \propto M^{3 / 2} / \varpi^{3 / 2} \propto \Sigma^{3 / 2} \varpi^{3 / 2} \propto\left(1+H_{0}\right)^{3 / 2}$. To reconcile this result with the correct value, we require

$$
\phi_{*} \simeq \frac{\phi_{* \text { non }}}{\left(1+H_{0}\right)^{1 / 2}} .
$$

2 MP97 assumed that the gas was isentropic - i.e., that the adiabatic index $\gamma$ is the same as the polytropic index $\gamma_{p}$. As discussed above, the large observed density contrasts in molecular clouds suggest that $\gamma>\gamma_{p}$. No calculations are available for this case, however, so we have adopted the isentropic results of MP97. 
Li \& Shu regard $H_{0} \sim 1$ as typical for low-mass star formation, which would lead to a reduction in the accretion rate by a factor of about 1.4.

The star formation time $t_{* f}$ increases from $1.5\left(1+H_{0}\right)^{1 / 2} t_{\mathrm{ff}, s}$ to $3.5\left(1+H_{0}\right)^{1 / 2} t_{\mathrm{ff}, s}$ as $\gamma_{p}$ decreases from 1 to 0 ; for the fiducial case, $t_{* f}=2.22\left(1+H_{0}\right)^{1 / 2} t_{\mathrm{ff}, s}$. (Note, however, that models with $\gamma_{p}$ close to zero are not realistic because they have no pressure gradient: $k_{P} \rightarrow 0$ from eq. 2. Also, see the discussion of logatropes in Appendix B). The ratio of the star formation time to the mean free-fall time of the core, $\bar{t}_{\mathrm{ff}}$, core $\equiv\left(3 \pi / 32 G \bar{\rho}_{\text {core }}\right)^{1 / 2}$, is larger by a factor $\left(\bar{\rho}_{\text {core }} / \rho_{s}\right)^{1 / 2}=\left[3 /\left(3-k_{\rho}\right)\right]^{1 / 2}$. For example, for $k_{\rho}=1.5$, we have $t_{* f}=3.14\left(1+H_{0}\right)^{1 / 2} \bar{t}_{\mathrm{ff}}$, core. Finally, we note that if we use equation (25) to normalize the star formation time to the mean free-fall time of the clump in which the core is embedded, we find

$$
t_{* f}=0.98\left(\frac{1+H_{0}}{2}\right)^{1 / 2}\left(\frac{m_{* f}}{30 M_{\odot}}\right)^{1 / 4}\left(\frac{4000 M_{\odot}}{M_{\mathrm{cl}}}\right)^{1 / 4} \bar{t}_{\mathrm{ff}, \mathrm{cl}} .
$$

Hence, for typical conditions in Galactic regions of high-mass star formation, the time to form a star is about equal to the mean free-fall time of the parent clump, $t_{* f} \simeq t_{\mathrm{ff}, \mathrm{cl}}$. In super star clusters, which have $M_{\mathrm{cl}} \sim 10^{6} M_{\odot}$, the star formation time is somewhat less than this.

\subsection{Evaluation of the Protostellar Accretion Rate and Star Formation Timescale}

The general expression for the accretion rate in terms of the surface pressure and the final stellar mass can now be inferred from equations (31)-(33):

$$
\dot{m}_{*}=\phi_{*} \frac{m_{* f}}{t_{\mathrm{ff}, s}}\left(\frac{m_{*}}{m_{* f}}\right)^{j}
$$

where

$$
j \equiv \frac{3\left(2-k_{\rho}\right)}{2\left(3-k_{\rho}\right)} .
$$

In the fiducial case $\left(k_{\rho}=1.5\right)$, we have $j=0.5$; for $k_{\rho}=1.8$, we have $j=0.25$. Using equation (8) to evaluate $t_{\mathrm{ff}, s} \propto\left(1 / \rho_{\text {core }, s}\right)^{1 / 2}$, we find

$$
\begin{aligned}
\dot{m}_{*} & =9.53 \times 10^{-4} \phi_{*} A^{1 / 8} k_{P}^{1 / 4} \epsilon_{\text {core }}^{1 / 4}\left(\frac{m_{* f}}{30 M_{\odot}}\right)^{3 / 4}\left(\frac{P_{s, \text { core }} / k}{10^{9} \mathrm{~K} \mathrm{~cm}^{-3}}\right)^{3 / 8}\left(\frac{m_{*}}{m_{* f}}\right)^{j} M_{\odot} \mathrm{yr}^{-1}, \\
& \rightarrow 4.6 \times 10^{-4}\left(\frac{m_{* f}}{30 M_{\odot}}\right)^{3 / 4} \Sigma_{\mathrm{cl}}^{3 / 4}\left(\frac{m_{*}}{m_{* f}}\right)^{0.5} M_{\odot} \mathrm{yr}^{-1},
\end{aligned}
$$

where again we have used the symbol $\rightarrow$ to emphasize that the final expression applies only in the fiducial case. Although

\begin{tabular}{|c|c|c|}
\hline Parameter & Definition & Fiducial Value \\
\hline$A$ & $\left(3-k_{\rho}\right)\left(k_{\rho}-1\right) f_{q}$ & $1 / 2$ (clumps), $3 / 4$ (cores) \\
\hline$f_{\text {acc }}$ & fraction of core's gravitational energy radiated by star+disk & 0.5 \\
\hline$f_{g}$ & gas mass fraction: $M_{\mathrm{g}} / M=\Sigma_{\mathrm{g}} / \Sigma$ & $2 / 3$ (clumps), 1 (cores) \\
\hline$H_{0}$ & magnetic field parameter in Li-Shu cores & 1.0 \\
\hline$k_{\rho}$ & clump and core density structure: $\rho \propto r^{-k_{\rho}}$ & $3 / 2$ \\
\hline$k_{P}$ & clump and core pressure structure: $P \propto r^{-k_{P}}$ & $2\left(k_{\rho}-1\right)=1$ \\
\hline$m_{A}$ & Alfven Mach number & 1.0 \\
\hline$\alpha_{\text {vir }}$ & virial parameter, $5\left\langle\sigma^{2}\right\rangle R /(G M)$ & 1.0 \\
\hline$\epsilon_{\text {core }}$ & core star formation efficiency & 0.5 \\
\hline$\gamma_{p}$ & polytropic index: $P \propto \rho^{\gamma_{p}}$ & $k_{P} / k_{\rho}=2 / 3$ \\
\hline$\phi_{\text {geom }}$ & deviation from sphericity: $R_{m}^{3} /\left(R^{2} Z\right)$ & 1.0 \\
\hline$\phi_{B}$ & $\left\langle c^{2}\right\rangle /\left\langle\sigma^{2}\right\rangle=1.3+3 /\left(2 m_{A}^{2}\right)$ & 2.8 \\
\hline$\phi_{\bar{P}}$ & $\overline{P_{\mathrm{cl}}} /\left(G \Sigma_{\mathrm{cl}}^{2}\right)=(3 \pi / 20) f_{g} \phi_{\text {geom }} \phi_{B} \alpha_{\mathrm{vir}}$ & 0.88 \\
\hline$\phi_{P, \text { core }}$ & $P_{\mathrm{cl}}(r) / \bar{P}_{\mathrm{cl}}$ & 2.0 \\
\hline$\phi_{\rho}$, core & $\rho_{\mathrm{cl}}(r) / \bar{\rho}_{\mathrm{cl}}$ & 2.6 \\
\hline$\phi_{* \text { non }}$ & $\dot{m}_{*} t_{\mathrm{ff}} / m_{*} \quad$ (non-magnetic case) & 0.90 \\
\hline$\phi_{*}$ & $\phi_{* \text { non }} /\left(1+H_{0}\right)^{1 / 2}$ & 0.64 \\
\hline
\end{tabular}
the exponent $j$ in the relation $\dot{m}_{*} \propto m_{*}^{j}$ changes with $k_{\rho}$, the numerical coefficient is constant to within $10 \%$ for $1.3<$ $k_{\rho}<2$. The evolution of the mass-accretion rate and the mass is illustrated in Figure 2. Observe that our expression

TABLE 2

MODEL PARAMETERS AND THEIR FIDUCIAL VALUES 


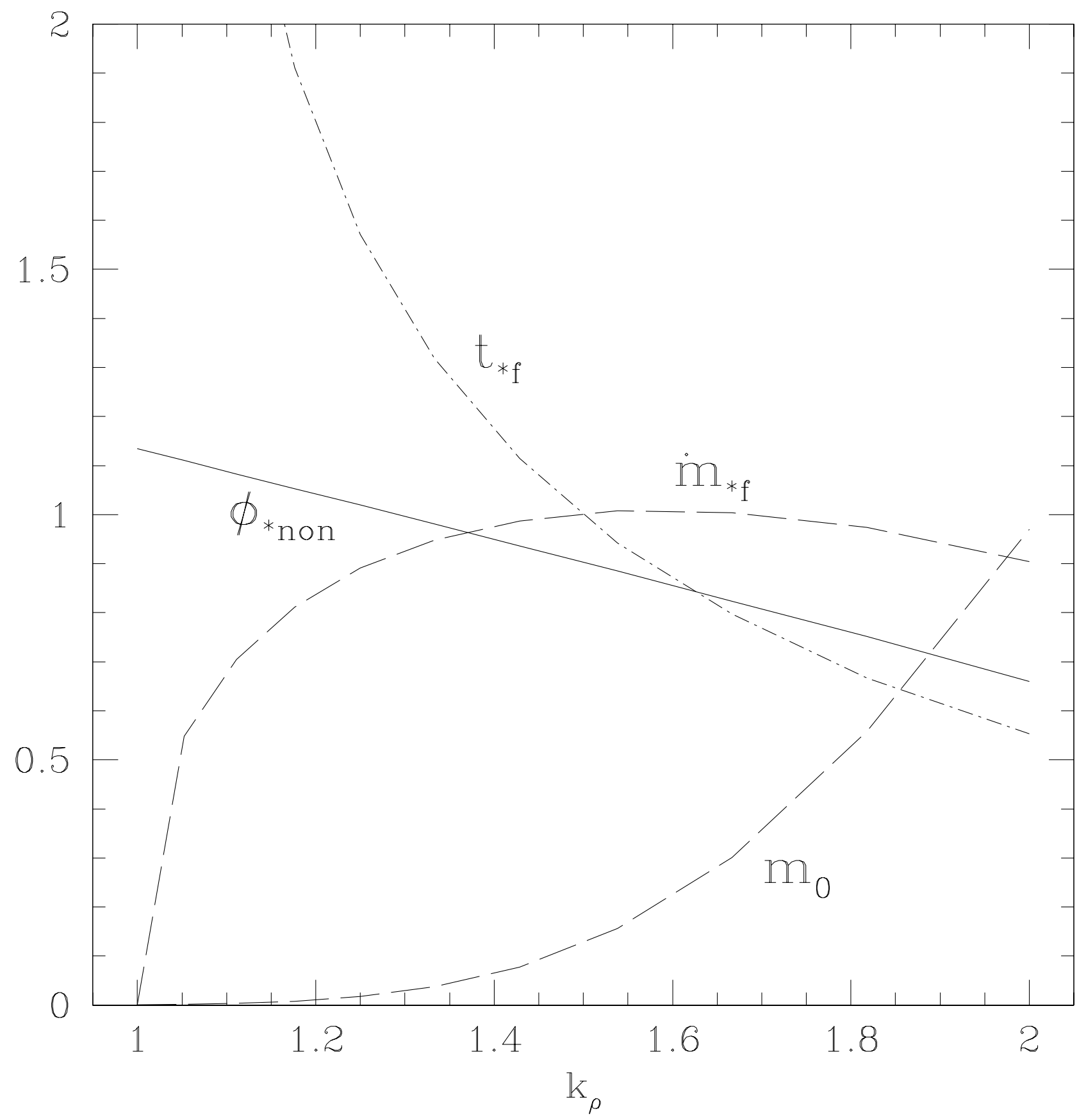

FIG. 1.- Variation of model parameters $\left(m_{0}, \phi_{* \text { non }}\right)$ and results $\left(\dot{m}_{* f}, t_{* f}\right)$ with $k_{\rho}$. Values of $m_{0}$ are from Table 4 of MP97. Over the entire range of $\gamma_{p}$ and $k_{\rho}$ relevant to molecular clouds $\left(0 \leq \gamma_{p} \leq 1,1 \leq k_{\rho} \leq 2\right), \phi_{* \text { non }} \simeq 1.62-0.48 k_{\rho}$ to within about $1 \%$. The star formation time decreases from $3.5 t_{\mathrm{ff}}$ to $1.5 t_{\mathrm{ff}}$ as $\gamma_{p}$ varies from 0 to 1 . The variation of $\dot{m}_{* f}$ and $t_{* f}$ relative to the $k_{\rho}=1.5$ case is also shown. Note that the singular polytropic model in hydrostatic equilibrium breaks down for $k_{\rho}=1, \gamma_{p}=0$, since then the pressure gradient vanishes $\left(k_{P}=0\right)$. 
for the accretion rate depends on two dimensional parameters, the final stellar mass, $m_{* f}$, and the surface density of the clump, $\Sigma_{\mathrm{cl}}$ (or, equivalently, the pressure in the clump), as well as on the ratio of the current stellar mass, $m_{*}$, to the final. We have expressed our results in this form in order to facilitate comparison with observation. In fact, the accretion rate depends on only two parameters, the enclosed mass, $M$, and the parameter $K_{p} \equiv P / \rho^{\gamma_{p}}$, which is constant for polytropes (Tan \& McKee in preparation).

The fiducial case includes the effect of a magnetic field. How does the field affect the accretion rate? From equations (10), (13), and (38) we have

$$
\dot{m}_{*} \propto \phi_{*} P_{s, \text { core }}^{3 / 8} \propto \phi_{*} \phi_{\bar{P}}^{3 / 8} \propto \frac{\phi_{B}^{3 / 8}}{\left(1+H_{0}\right)^{1 / 2}} \rightarrow 1.04
$$

since $\phi_{B}=2.8$ and $H_{0}=1$ in the fiducial case. There is therefore very little difference between this case and the field-free case considered by MT. It should be kept in mind that we have assumed that the value of the virial parameter $\alpha_{\text {vir }}$ has been taken from observation and is therefore fixed as the field is varied. As discussed in Appendix A, if the value of the virial parameter is taken from theory, then $\alpha_{\text {vir }} \phi_{B}$ is fixed and the accretion rate would vary as $\left(1+H_{0}\right)^{-1 / 2}$.

The corresponding value of the star-formation time is

$$
\begin{aligned}
t_{* f} & =3.15 \times 10^{4}\left(\frac{4-3 \gamma_{p}}{\phi_{*} A^{1 / 8} k_{P}^{1 / 4} \epsilon_{\text {core }}^{1 / 4}}\right)\left(\frac{m_{* f}}{30 M_{\odot}}\right)^{1 / 4}\left(\frac{10^{9} \mathrm{~K} \mathrm{~cm}^{-3}}{P_{s, \text { core }} / k}\right)^{3 / 8} \mathrm{yr}, \\
& \rightarrow 1.29 \times 10^{5}\left(\frac{m_{* f}}{30 M_{\odot}}\right)^{1 / 4} \Sigma_{\mathrm{cl}}^{-3 / 4} \mathrm{yr} .
\end{aligned}
$$

For example, with the fiducial values of our parameters, a $100 M_{\odot}$ star forming in a clump with $\Sigma_{\mathrm{cl}}=1 \mathrm{~g} \mathrm{~cm}^{-2}$ has a final accretion rate of $1.1 \times 10^{-3} M_{\odot} \mathrm{yr}^{-1}$ and a star-formation time of $1.74 \times 10^{5} \mathrm{yr}$. If the value $\Sigma_{\mathrm{cl}} \simeq 0.24 \mathrm{~g} \mathrm{~cm} \mathrm{~cm}^{-2}$ inferred for the Orion Nebula Cluster was appropriate for the molecular gas out of which the Trapezium stars formed, then the time scale to form a $30 M_{\odot}$ star in Orion was about $3.8 \times 10^{5} \mathrm{yr}$. The dependence of $t_{* f}$ on $k_{\rho}$ is shown in Figure 1 . For example, had we adopted the value of $k_{\rho}=1.8$ found by Mueller et al. $(2002 \mathrm{~b})$, the coefficient in equation (44) would have dropped to $8.9 \times 10^{4} \mathrm{yr}$. The star-formation time as a function of final stellar mass is shown in Figure 3 . Effects of changing the pressure and using a different, though still representative, value of $k_{\rho}$ are also shown.

Our results for the accretion rate and star formation time have been presented in terms of the surface density of the star-forming clump. However, if higher resolution observations are available, then it is convenient to express these results in terms of the measured density profile, $n_{\mathrm{H}}=n_{\mathrm{H}, \text { ref }}\left(r / r_{\text {ref }}\right)^{-k_{\rho}}$. Note that the resolution must be high enough so that the data apply to the core forming an individual star, and not to the clump in which many stars are forming; for the fiducial case, this requires measuring the structure on a scale less than about 0.05 pc $~ 10^{4}$ AU (eq. 19). Expressing the density in terms of the mass, we have

$$
n_{\mathrm{H}}=\left[\frac{4 \pi \mu_{\mathrm{H}} n_{\mathrm{H}, \mathrm{ref}}^{3 / k_{\rho}} r_{\mathrm{ref}}^{3}}{\left(3-k_{\rho}\right) M}\right]^{k_{\rho} /\left(3-k_{\rho}\right)},
$$

where $\mu_{\mathrm{H}}=2.34 \times 10^{-24} \mathrm{~g}$ is the mean mass per $\mathrm{H}$. We now ask, how long will it take the gas in $M$ to form a star of mass $\epsilon_{\text {core }} M$ ? Equation (33) yields

$$
\begin{aligned}
t_{* f} & =\frac{1}{\phi_{*} k_{\rho}}\left(\frac{3 \pi}{8 G}\right)^{1 / 2}\left[\frac{\left(3-k_{\rho}\right)^{6-k_{\rho}}}{\left(n_{\mathrm{H}, \mathrm{ref}} \mu_{\mathrm{H}}\right)^{3}}\left(\frac{m_{*}}{4 \pi \epsilon_{\mathrm{core}} r_{\mathrm{ref}}^{3}}\right)^{k_{\rho}}\right]^{1 / 2\left(3-k_{\rho}\right)} \\
& \rightarrow 5.79 \times 10^{4}\left(\frac{10^{8} \mathrm{~cm}^{-3}}{n_{\mathrm{H}, \mathrm{ref}}}\right)\left(\frac{1000 \mathrm{AU}}{r_{\mathrm{ref}}}\right)^{3 / 2}\left(\frac{m_{*}}{30 M_{\odot}}\right)^{1 / 2} \mathrm{yr} .
\end{aligned}
$$

Had we chosen $k_{\rho}=1.8$, the typical value found by Mueller et al. (2002b), the result is only slightly different:

$$
t_{* f} \rightarrow 8.07 \times 10^{4}\left(\frac{10^{8} \mathrm{~cm}^{-3}}{n_{\mathrm{H}, \text { ref }}}\right)^{5 / 4}\left(\frac{1000 \mathrm{AU}}{r_{\text {ref }}}\right)^{9 / 4}\left(\frac{m_{*}}{30 M_{\odot}}\right)^{3 / 4} \quad \mathrm{yr} .
$$

The median value of $n_{\mathrm{H} \text {, ref }}$ at $r_{\text {ref }}=10^{3} \mathrm{AU}$ in the Mueller et al. sample is $2.4 \times 10^{7} \mathrm{~cm}^{-3}$ (based on the Ossenkopf \& Henning 1994 opacities), which gives $t_{* f} \simeq 5 \times 10^{5}$ yr for a $30 M_{\odot}$ star. Recall that this choice of dust opacity led to masses that averaged 3.4 times less than the virial mass; if the clouds were at their virial masses, the densities would be 3.4 times higher and the median time to form a $30 M_{\odot}$ star would be $10^{5} \mathrm{yr}$.

Recently, Doty et al. (2002) have estimated the timescale for massive star formation using chemical clocks. From an analysis of the source AFGL 2591, they infer that the chemical timescale is in the range $7 \times 10^{3}-5 \times 10^{4}$ yr, with a preference for $3 \times 10^{4} \mathrm{yr}$. The protostellar models discussed in $\S 6$ below suggest that the central star has a mass of about $13 M_{\odot}$. The time scale measured by Doty et al. refers only to a part of the star formation time, since the chemistry is strongly affected by the rapidly rising luminosity. The time scale they infer is in qualitative agreement with our results. Unfortunately, our results do not apply to the the case $k_{\rho}=1$ they used to model their data, since such a model cannot be in a self-gravitating equilibrium (see Appendix B); as a result, we cannot make a quantitative comparison. 
Henriksen (private communication) has pointed out that the $m_{* f}^{1 / 4}$ dependence of the star-formation timescale was presented in Henriksen \& Turner (1984) and Henriksen (1986). This scaling is to be expected, since it follows directly from the assumption of hydrostatic equilibrium. In these models it was assumed that stars form when the turbulence becomes sub-sonic and that the pressure is a constant in GMCs. As we have seen, the former assumption is incorrect for massive stars, and the latter assumption leads to a significant over-estimate of the star-formation time for massive stars.

Adams (private communication) has pointed out that results similar to ours are implicit in Adams and Fatuzzo (1996). By dividing their estimate for the mass of a protostar by the accretion rate $c^{3} / G$, one finds that the star formation times are about $10^{5} \mathrm{yr}$ and depend only weakly on the mass. Their work is based on the assumptions that (1) protostellar accretion is halted when the rate at which mass falls directly onto the star, rather than through a disk, is the same as the mass loss rate in the protostellar outflow; and (2) that the wind luminosity is a fixed fraction of the stellar luminosity. The validity of these assumptions, particularly for massive star formation, remains to be established.

\subsection{Turbulent Core Accretion vs. Competitive Accretion}

An alternative model for massive star formation is the competitive accretion model, in which massive stars form by accreting gas that was initially not gravitationally bound to the star. The accreting stars compete for the mass available in the clump, and it has been suggested that this process leads to the IMF (Bonnell et al. 1997; Bonnell et al. 2001). By contrast, the turbulent core model is based on the premise that most of the gas that goes into a star was initially bound. Since the core is turbulent and is embedded in a turbulent medium, this is undoubtedly an oversimplification - at the very least, some of the gas that was bound to the core at the onset of star formation will become unbound during the course of the star formation, and similarly some gas that was initially unbound will become bound. First we estimate the amount of mass exchange between the core and the clump, and then consider the rate of accretion directly onto a star once it has formed from its core.

In general, accretion occurs at a rate given by

$$
\dot{m}_{\mathrm{acc}}=\pi \rho_{\mathrm{cl}} v_{\mathrm{rel}} R_{\mathrm{acc}}^{2}
$$

where $\rho_{\mathrm{cl}}=\phi_{\rho, \text { core }} \bar{\rho}_{\mathrm{cl}}$ is the density of the ambient clump material, $v_{\mathrm{rel}}$ is the relative velocity between this material and the accreting object, be it core or star, and $R_{\text {acc }}$ is the accretion radius, which is the largest possible impact parameter of approaching gas that ends up bound to the core or star. Recall that the factor $\phi_{\rho \text {, core }} \simeq 2.6$ (§3.2) allows for the central concentration of clump density distribution and massive star formation. We assume that the relative velocity of the core or star and the ambient medium is $\sqrt{ } 3 \sigma_{\mathrm{cl}}=\sqrt{ } 3\left(\phi_{P, \text { core }} / \phi_{\rho, \text { core }}\right)^{1 / 2}\left\langle\sigma_{\mathrm{cl}}^{2}\right\rangle$. The rms velocity dispersion in the clump, $\left\langle\sigma_{\mathrm{cl}}^{2}\right\rangle$, is related to the virial parameter $\alpha_{\mathrm{vir}}$ by equation (A3), under the assumption that the clump is spherical. We then find

$$
v_{\text {rel }}=\sqrt{ } 3\left(\frac{\phi_{P, \text { core }}}{\phi_{\rho, \text { core }}}\right)^{1 / 2}\left\langle\sigma_{\mathrm{cl}}^{2}\right\rangle^{1 / 2}=4.47\left(\frac{\phi_{P, \text { core }}}{\phi_{\rho, \text { core }}}\right)^{1 / 2} \Sigma^{1 / 4} \alpha_{\text {vir }}^{1 / 2}\left(\frac{M_{\mathrm{cl}}}{4000 M_{\odot}}\right)^{1 / 4} \mathrm{~km} \mathrm{~s}^{-1}
$$

\subsubsection{Accretion by the core}

Equation (37) shows that in typical Galactic star-forming clumps, the star formation time is comparable to the free-fall time of the clump, which in turn is comparable to the time for the core to cross the clump. Since the surface density of the core and the clump are similar (see eq. 23) we anticipate that the core will interact with a mass comparable to its own as the star forms, or equivalently the rate at which a core interacts with clump mass should be similar to the rate of star formation.

To make this idea more quantitative, we estimate $R_{\text {acc }}$ for the core by considering particle orbits that intercept its surface. In this case $R_{\text {acc }}=\left(1+v_{\text {esc }}^{2} / v_{\text {rel }}^{2}\right)^{1 / 2} R_{\text {core }}$, where $v_{\text {esc }}$ is the escape velocity from the core,

$$
\begin{aligned}
v_{\mathrm{esc}} & =2.27\left(\frac{m_{* f}}{30 M_{\odot}}\right)^{1 / 4} \epsilon_{\mathrm{core}}^{-1 / 4}\left(\frac{k_{P}^{2} \phi_{P, \text { core }} \phi_{\bar{P}}}{A}\right)^{1 / 8} \Sigma_{\mathrm{cl}}^{1 / 4} \mathrm{~km} \mathrm{~s}^{-1}, \\
& \rightarrow 3.00\left(\frac{m_{* f}}{30 M_{\odot}}\right)^{1 / 4} \Sigma_{\mathrm{cl}}^{1 / 4} \mathrm{~km} \mathrm{~s}^{-1}
\end{aligned}
$$

The gravitational enhancement of the geometric cross-section is thus

$$
\phi_{\text {grav }} \rightarrow 1+0.59 \alpha_{\text {vir }}^{-1}\left(\frac{m_{* f}}{30 M_{\odot}}\right)^{1 / 2}\left(\frac{M_{\mathrm{cl}}}{4000 M_{\odot}}\right)^{-1 / 2}
$$

The enhancement is significant only for massive cores in relatively low mass clumps. The rate of accretion is thus

$$
\begin{aligned}
\dot{m}_{\mathrm{acc}} & =2.50 \times 10^{-4}\left(\frac{A \phi_{\rho, \text { core }} f_{g}^{2} \alpha_{\mathrm{vir}} \phi_{\text {grav }}^{2}}{k_{P}^{2} \epsilon_{\mathrm{core}}^{2} \phi_{\bar{P}}}\right)^{1 / 2}\left(\frac{m_{* f}}{30 M_{\odot}}\right)\left(\frac{M_{\mathrm{cl}}}{4000 M_{\odot}}\right)^{-1 / 4} \Sigma_{\mathrm{cl}}^{3 / 4} M_{\odot} \mathrm{yr}^{-1}, \\
& \rightarrow 7.9 \times 10^{-4}\left(\frac{\phi_{\text {grav }}}{1.6}\right)\left(\frac{m_{* f}}{30 M_{\odot}}\right)\left(\frac{M_{\mathrm{cl}}}{4000 M_{\odot}}\right)^{-1 / 4} \Sigma_{\mathrm{cl}}^{3 / 4} M_{\odot} \mathrm{yr}^{-1} .
\end{aligned}
$$

Thus we see that a massive core will tend to interact with clump material at a rate that is comparable to the rate at which it is collapsing or being eroded by star formation, $\dot{m}_{*} / \epsilon_{\text {core. }}$. This rate is a factor of several smaller in super star clusters with $M_{\mathrm{cl}} \sim 10^{6} M_{\odot}$. 
What is the fate of this swept-up mass? Gas between the cores ("intercore gas") is likely to have an Alfven velocity that is greater than the Keplerian velocity at the surface of the core in view of its low density; it will therefore not be bound to the core after being swept up. It is likely that most of the mass is in cores, however, and insofar as the core mass distribution follows the IMF, most of this mass will be in low-mass cores. When one of these cores strikes the outer region of a massive core, it is quite likely that it will strip gas from the massive core, since the relative velocities are $\gtrsim v_{\text {esc }}$. On the other hand, if it strikes the inner parts of the massive core, it is likely to be absorbed by the larger core. Accretion of ambient material onto the massive core will decrease as soon as a protostellar outflow forms, and it will decrease further when the protostar becomes massive enough to generate significant radiation pressure. Thus, the interaction of the core with the ambient clump will result in an exchange of material that most likely causes the massive core to grow, but at a smaller rate than suggested by equation (55). In fact, this growth may be the mechanism that causes an initially bound, but stable, core to evolve into an unstable core that collapses to form a star. This growth will not be a runaway process since $R_{\text {core }}$ cannot grow significantly once the expansion wave reaches the edge of the core.

\subsubsection{Bondi-Hoyle accretion by the star}

In the case of competitive accretion, it is the protostar that accretes gas from the clump. In this case, the appropriate accretion radius is given by $R_{\mathrm{acc}}=R_{\mathrm{BH}} \simeq 2 G m_{*} /\left(v_{\mathrm{rel}}^{2}+c^{2}\right)$ (Bondi \& Hoyle 1944). We generalize this result by including the effects of magnetic fields, $c^{2}=\phi_{B} \sigma^{2}$, so that

$$
R_{\mathrm{BH}}=\left(\frac{\phi_{\rho, \text { core }} / \phi_{P, \text { core }}}{3+\phi_{B}}\right) \frac{2 G m_{*}}{\left\langle\sigma_{\mathrm{cl}}^{2}\right\rangle}
$$

Evaluating this expression in terms of the virial parameter $\alpha_{\text {vir }}$, we obtain

$$
\begin{aligned}
R_{\mathrm{BH}} & =\frac{10}{\alpha_{\mathrm{vir}}}\left(\frac{\phi_{\rho, \text { core }} / \phi_{P, \text { core }}}{3+\phi_{B}}\right)\left(\frac{m_{*}}{M_{\mathrm{cl}}}\right) R_{\mathrm{cl}} \\
& \rightarrow 2.89 \times 10^{-3} \alpha_{\mathrm{vir}}^{-1}\left(\frac{m_{*}}{10 M_{\odot}}\right)\left(\frac{M_{\mathrm{cl}}}{4000 M_{\odot}}\right)^{-1 / 2} \Sigma_{\mathrm{cl}}^{-1 / 2} \mathrm{pc},
\end{aligned}
$$

where we made use of the relation $R_{\mathrm{cl}}=\left(M_{\mathrm{cl}} / \pi \Sigma_{\mathrm{cl}}\right)^{1 / 2}=0.516\left(M_{\mathrm{cl}} / 4000 M_{\odot}\right)^{1 / 2} \Sigma_{\mathrm{cl}}^{-1 / 2}$ pc.

This estimate for the accretion radius is actually an upper limit for two reasons: First, it is based on the assumption that the accreting gas is of uniform density (on scales of $R_{\mathrm{BH}}$ ) so that it can efficiently dissipate in the wake of the star. Second, Bondi-Hoyle accretion will cease once the stellar mass is $\gtrsim 10 M_{\odot}$, since then the main sequence radiation pressure is enough to counter the star's gravitational attraction, thereby preventing the focusing of gas streamlines in the wake of the star (Bonnell et al. 1998). We therefore normalize our expressions to a stellar mass of $10 M_{\odot}$ in this subsection.

With this result for the accretion radius, we find that the Bondi-Hoyle accretion rate in a star-forming clump is

$$
\begin{aligned}
\dot{m}_{*, \mathrm{BH}} & =1.66 \times 10^{-5}\left[\frac{f_{\mathrm{g}} \phi_{\rho}^{5 / 2} \text {, core }}{\alpha_{\mathrm{vir}}^{3 / 2} \phi_{P, \text { core }}^{3 / 2}\left(3+\phi_{B}\right)^{2}}\right]\left(\frac{m_{*}}{10 M_{\odot}}\right)^{2}\left(\frac{M_{\mathrm{cl}}}{4000 M_{\odot}}\right)^{-5 / 4} \Sigma_{\mathrm{cl}}^{3 / 4} M_{\odot} \mathrm{yr}^{-1}, \\
& \rightarrow 1.27 \times 10^{-6} \alpha_{\mathrm{vir}}^{-3 / 2}\left(\frac{m_{*}}{10 M_{\odot}}\right)^{2}\left(\frac{M_{\mathrm{cl}}}{4000 M_{\odot}}\right)^{-5 / 4} \Sigma_{\mathrm{cl}}^{3 / 4} M_{\odot} \mathrm{yr}^{-1} .
\end{aligned}
$$

We see that the Bondi-Hoyle accretion rate (for a $10 M_{\odot}$ star, the maximum possible for $\mathrm{BH}$ accretion) is more than two orders of magnitude lower than the turbulent core accretion rate calculated in $\S 4.1$. If the clump survives for $\sim 1 \mathrm{Myr}$ while it forms a star cluster then the maximum fractional change in a star's mass is $\sim 0.1\left(m_{*} / 10 M_{\odot}\right)\left(M_{\mathrm{cl}} / 4000 M_{\odot}\right)^{-5 / 4} \Sigma_{\mathrm{cl}}^{-3 / 4}$, so this process will not significantly alter the stellar mass under typical conditions of massive star formation.

\section{TWO-COMPONENT CORES}

Cores in molecular clouds are usually observed to have central regions dominated by thermal motions and envelopes dominated by nonthermal motions. The presence of a significant thermal pressure component in the inner regions of cores breaks the self-similarity of the structure observed on larger scales in molecular clouds (Williams et al. 2000). As discussed in $\S 3$, the nonthermal pressure component is modeled with $\gamma_{p}<1$ (Maloney 1988). Two components can be modeled as a multipressure polytrope in which $P=P_{\mathrm{th}}+P_{\mathrm{nth}}$, where $P_{\mathrm{th}} \equiv \rho c_{\mathrm{th}}^{2}$ is the thermal pressure and $P_{\mathrm{nth}}$ is the nonthermal pressure (McKee \& Holliman 1999). To allow for damping of nonthermal motions on small scales, the gas core can also be modeled as a composite polytrope, in which the central region is thermally supported and the envelope is supported by both thermal and nonthermal motions (Curry \& McKee 2000). For analytic modeling, however, the simple "TNT" (thermal plus nonthermal) model introduced by Myers \& Fuller (1992) and developed by Caselli \& Myers (1995) is convenient. In this model, the density is assumed to be of the form

$$
\rho \propto\left(\frac{r_{0}}{r}\right)^{k_{\rho}}+\left(\frac{r_{0}}{r}\right)^{2}
$$

with $1<k_{\rho}<2$. We assume that $\left(R_{\text {core }} / r_{0}\right)^{2-k_{\rho}} \gg 1$, so that the first term dominates at the surface of the core and the model is equivalent to the sum of a singular polytropic sphere and a singular isothermal sphere:

$$
\rho=\rho_{s}\left(\frac{R_{\text {core }}}{r}\right)^{k_{\rho}}+\frac{c_{\mathrm{th}}^{2}}{2 \pi G r^{2}},
$$




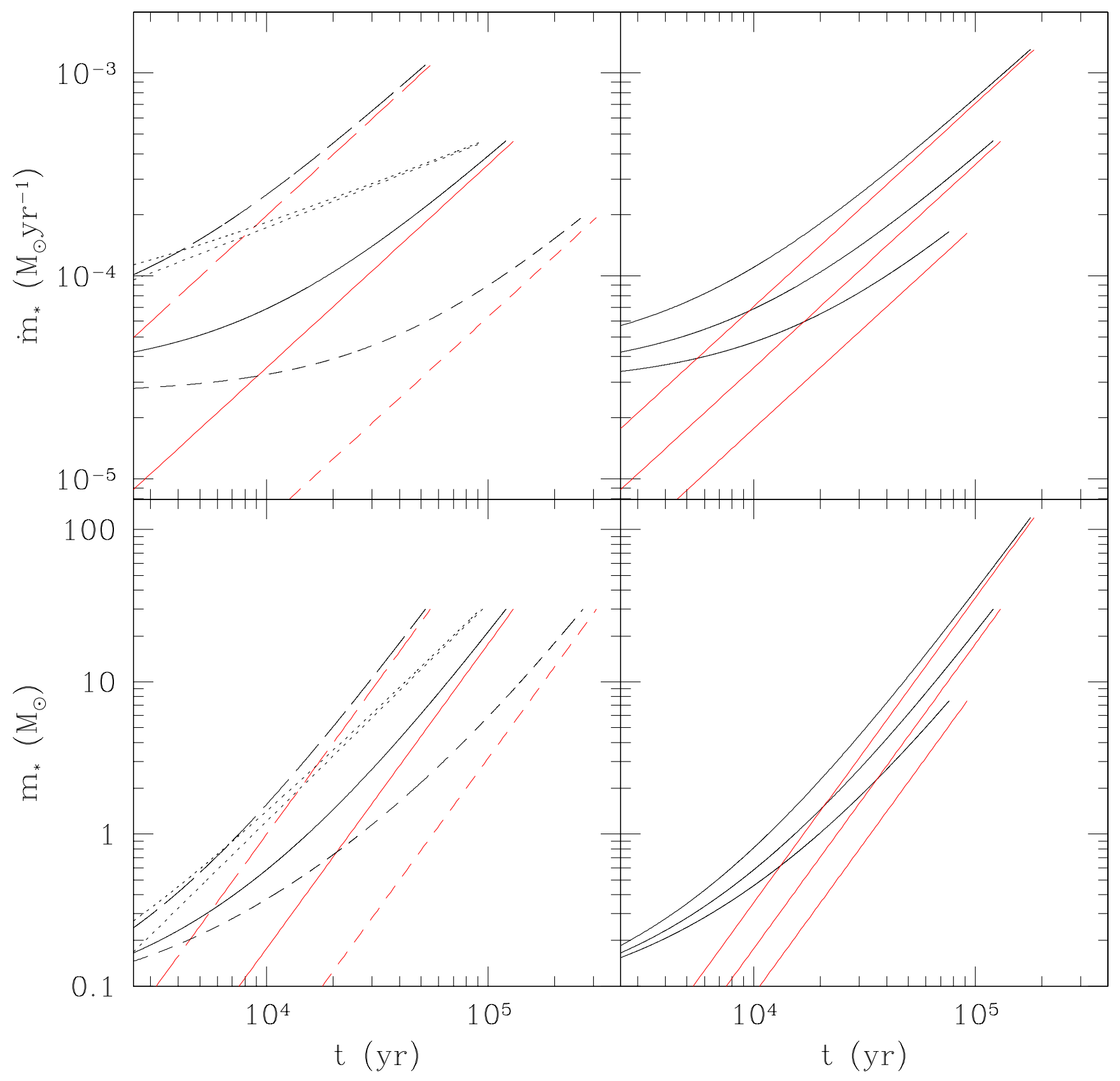

Fig. 2.- Evolution of $\dot{m}_{*}$ (top panels) and $m_{*}$ (bottom panels). The straight lines show the purely nonthermal model, while the curved lines show the two component (thermal $[100 \mathrm{~K}]+$ nonthermal) core model described in $\S 5$. The left panels show only $m_{* f}=30 M_{\odot}$ models. The different cases are $k_{\rho}=1.5$ with $\Sigma_{\mathrm{cl}}=0.316,1.0,3.16 \mathrm{~g} \mathrm{~cm}^{-2}$ (short-dashed, solid, long-dashed, respectively), and $k_{\rho}=1.75$ with $\Sigma_{\mathrm{cl}}=1 \mathrm{~g} \mathrm{~cm}^{-2}$ (dotted line). The right panels show cases with $m_{* f}=7.5,30,120 M_{\odot}$ (bottom to top, respectively) for $\Sigma_{\mathrm{cl}}=1 \mathrm{~g} \mathrm{~cm}^{-2}$ and $k_{\rho}=1.5$. 


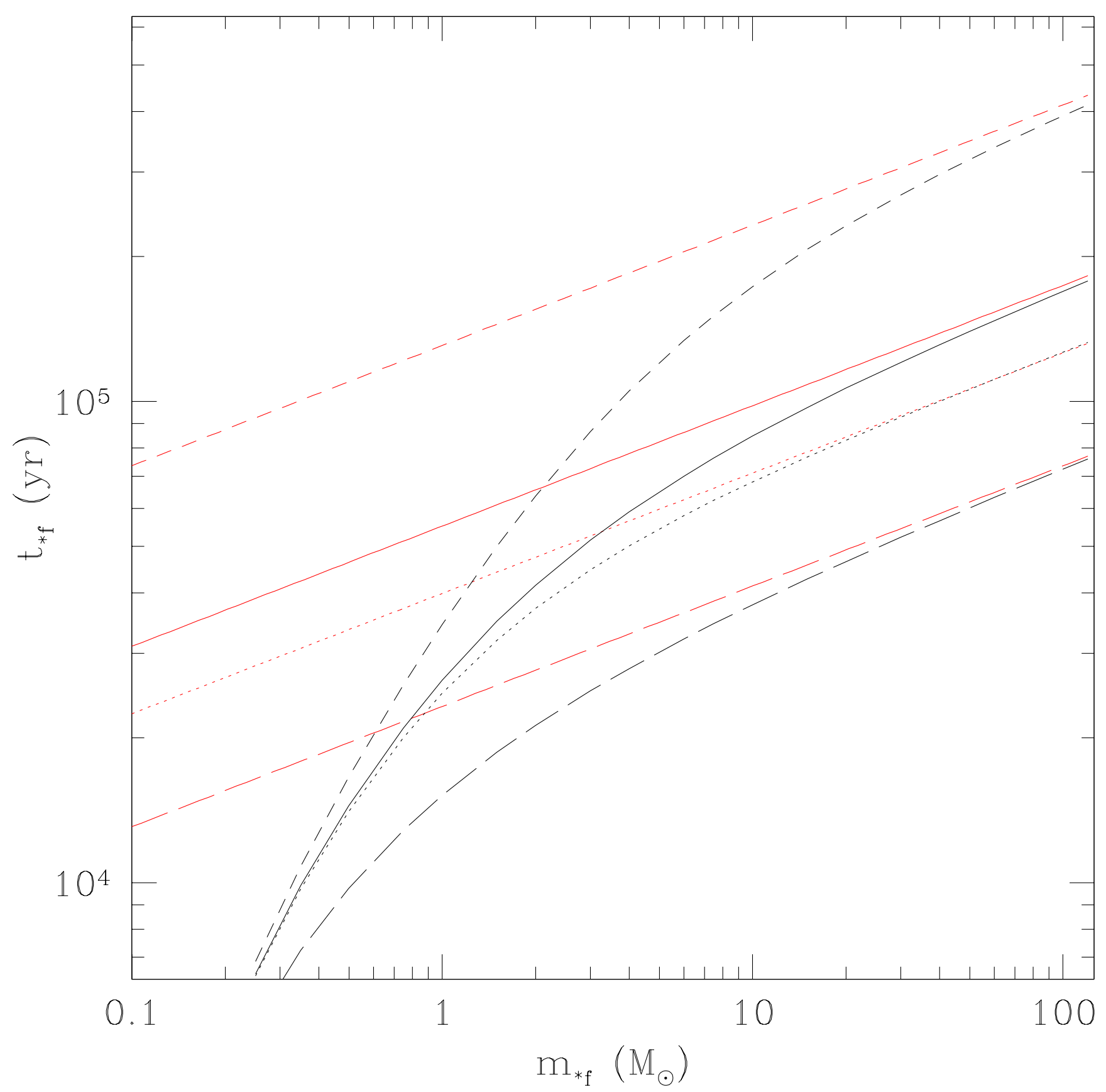

FIG. 3.- Star formation time $t_{* f}$ versus final stellar mass $m_{* f}$ for accretion from cores with $k_{\rho}=1.5$ at $\Sigma_{\mathrm{cl}}=0.316,1,3.16 \mathrm{~g} \mathrm{~cm}{ }^{-2}$ (short-dashed, solid, long-dashed lines) and with $k_{\rho}=1.75$ at $\Sigma_{\mathrm{cl}}=1 \mathrm{~g} \mathrm{~cm}^{-2}$ (dotted line). As in Figure 2, the straight lines show the purely nonthermal model, while the curved lines show the two component (thermal $[100 \mathrm{~K}]+$ nonthermal) core model described in $\S 5$. 
where, by construction, $\rho_{s}$ is very nearly equal to the density at the surface; it is therefore given by equation (8).

To determine the mass-accretion rate $\dot{m}_{*}$ and the star-formation time $t_{* f}$, we need to know the value of the free-fall time, $t_{\mathrm{ff}} \propto[\rho(m)]^{-1 / 2}$. Since the TNT model is just a rough approximation to the actual density distribution, we can use equation (3) to replace the TNT density distribution $\rho(r)$ with a TNT density distribution $\rho(M)$,

$$
\begin{aligned}
\rho & =\rho_{s}\left(\frac{M_{\text {core }}}{M}\right)^{2(1-j)}+\frac{2 c_{\mathrm{th}}^{6}}{\pi G^{3} M^{2}}, \\
& \equiv \rho_{s}\left(\frac{M_{\text {core }}}{M}\right)^{2(1-j)}\left[1+\left(\frac{M_{\mathrm{th}}}{M}\right)^{2 j}\right],
\end{aligned}
$$

where $j$ is defined in equation (39). Here $M_{\text {th }}$ is the mass below which the thermal density distribution dominates, and it will be evaluated numerically below. ${ }^{3}$

The protostellar accretion rate is

$$
\dot{m}_{*}=\phi_{*} \frac{m_{*}}{t_{\mathrm{ff}}} \propto \frac{m_{*}}{t_{\mathrm{ff}, s}} \cdot \phi_{*}\left(\frac{\rho}{\rho_{s}}\right)^{1 / 2}
$$

from equation (28). Let $\phi_{* \text { th }}=0.975 \pi \sqrt{3} /\left[8\left(1+H_{0}\right)^{1 / 2}\right]$ be the value of $\phi_{*}$ for the isothermal component, and $\phi_{* \text { nth }}$ be the value for the nonthermal component. Generalizing equation (65) with the aid of equation (64) for the density of a two-component core, we find

$$
\dot{m}_{*} \simeq \dot{m}_{* f}\left[\left(\frac{m_{*}}{m_{* f}}\right)^{2 j}+\left(\frac{\phi_{* \text { th }}}{\phi_{* \text { nth }}}\right)^{2}\left(\frac{\epsilon_{\mathrm{core}} M_{\mathrm{th}}}{m_{* f}}\right)^{2 j}\right]^{1 / 2} .
$$

At early times, when the mass of the protostar is less than $\epsilon_{\mathrm{core}} M_{\mathrm{th}}$, the accretion rate is constant-it is given by the isothermal rate (eq. 1) corrected by a factor $\epsilon_{\text {core }} /\left(1+H_{0}\right)^{1 / 2}$ for the star formation efficiency and the effects of the magnetic field. Once the mass of the protostar exceeds this characteristic mass, the accretion rate rapidly approaches the nonthermal rate evaluated in $\S 4$. The evolution in time of the accretion rate and instantaneous stellar mass are shown in Figure 2 for two-component cores with a $T=100 \mathrm{~K}$ thermal core. The star formation time versus final stellar mass is shown in Figure 3.

We evaluate the mass of the thermal core, $M_{\mathrm{th}}$, in terms of the Bonnor-Ebert mass, which is the maximum mass of a stable isothermal sphere of gas (Bonnor 1956; Ebert 1955),

$$
\begin{aligned}
M_{\mathrm{BE}}=1.182 \frac{c_{\mathrm{th}}^{4}}{\left(G^{3} P_{s, \text { core }}\right)^{1 / 2}} & =0.0464\left(\frac{T}{20 \mathrm{~K}}\right)^{2}\left(\frac{10^{9} \mathrm{~K} \mathrm{~cm}^{-3}}{P_{s, \text { core }} / k}\right)^{1 / 2} M_{\odot}, \\
& \rightarrow 0.0504\left(\frac{T}{20 \mathrm{~K}}\right)^{2} \frac{1}{\Sigma_{\mathrm{cl}}} M_{\odot},
\end{aligned}
$$

where the numerical value is for a mean particle mass of $2.33 m_{\mathrm{H}}$. Equations (8) and (67) then imply

$$
\begin{aligned}
M_{\mathrm{th}} & =M_{\mathrm{BE}}\left(\frac{32}{1.182^{6} \pi^{3} A k_{P}^{2}}\right)^{\left(3-k_{\rho}\right) /\left[12\left(2-k_{\rho}\right)\right]}\left(\frac{M_{\mathrm{BE}}}{M_{f}}\right)^{\left(k_{\rho}-1\right) /\left[2\left(2-k_{\rho}\right)\right]}, \\
& \rightarrow 1.23 \times 10^{-3}\left(\frac{T}{20 \mathrm{~K}}\right)^{3}\left(\frac{30 \epsilon_{\mathrm{core}} M_{\odot}}{m_{* f}}\right)^{1 / 2} \frac{1}{\Sigma_{\mathrm{cl}}^{3 / 2}} M_{\odot} .
\end{aligned}
$$

If $M_{\text {core }}$ is comparable to $M_{\mathrm{BE}}$, then $M_{\mathrm{th}} \simeq M_{\mathrm{BE}}$; as $M_{\text {core }}$ increases, $M_{\mathrm{th}}$ decreases and becomes a negligible fraction of the core mass. Using equation (69), we can re-express the accretion rate as

$$
\dot{m}_{*} \simeq \dot{m}_{* f}\left[\left(\frac{m_{*}}{m_{* f}}\right)^{3\left(2-k_{\rho}\right) /\left(3-k_{\rho}\right)}+\left(\frac{\phi_{* \text { th }}}{\phi_{* \text { nth }}}\right)^{2}\left(\frac{32}{1.182^{6} \pi^{3} A k_{P}^{2}}\right)^{1 / 4}\left(\frac{\epsilon_{\mathrm{core}} M_{\mathrm{BE}}}{m_{* f}}\right)^{3 / 2}\right]^{1 / 2},
$$

which makes the dependence on $k_{\rho}$ clearer. This form of the accretion rate shows that the thermal term dominates throughout the accretion only if $m_{* f} \lesssim \epsilon_{\mathrm{core}} M_{\mathrm{BE}}$. In the fiducial case, the condition for the thermal term to dominate throughout is $m_{* f}<1.5 \times 10^{-2}(T / 20 \mathrm{~K})^{2} / \Sigma_{\mathrm{cl}} \mathrm{M}_{\odot}$; even for a temperature of $100 \mathrm{~K}$, this is only a fraction of a solar mass. We conclude that the thermal core is not important in the formation of stars with $m_{* f} \gtrsim 1 M_{\odot}$ in typical regions of massive star formation.

${ }^{3}$ It must be kept in mind that $M_{\mathrm{th}}$ is defined in terms of the density profile and not the sound speed. Evaluating $c^{2} / c_{\mathrm{th}}^{2}$ with the aid of equation (6), we find

$$
\frac{c^{2}}{c_{\mathrm{th}}^{2}}=\left(\frac{4}{A k_{P}^{2}}\right)^{1 / 3}\left(\frac{M}{M_{\mathrm{th}}}\right)^{2 j / 3} .
$$

For an isothermal gas $\left(\gamma_{p}=1, k_{\rho}=2\right.$ and $\left.j=0\right)$, we have $c^{2}=c_{\mathrm{th}}^{2}$ as expected. However, as $\gamma_{p}$ decreases below unity the value of $c^{2}$ at $M=M_{\mathrm{th}}$ increases above $c_{\mathrm{th}}^{2}$. Although the nonthermal pressure is greater than the thermal pressure at $M_{\mathrm{th}}$, the two pressure gradients are comparable there. 
Finally, we note that the fact that the pressure in regions of massive star formation is so high means that the minimum mass of an isolated star that can form is quite small,

$$
m_{* \min } \simeq \epsilon_{\mathrm{core}} M_{\mathrm{BE}} .
$$

If the temperature is of order $20 \mathrm{~K}$, as in the case of clustered low-mass star formation (Jijina, Myers, \& Adams 1999) and the pressure is that of a typical clump that is forming massive stars $\left(P_{s \text {, core }} \sim \bar{P}_{\mathrm{cl}} \sim 0.4 \times 10^{9} \mathrm{~K} \mathrm{~cm}^{-3}\right.$, corresponding to $\phi_{P \text {, core }}=1$ ), then this minimum mass is slightly less than $0.04 M_{\odot}$. As star formation proceeds in the clump, the temperature will rise and so will this typical minimum mass, $m_{* \min } \propto M_{\mathrm{BE}} \propto T^{2}$. On the other hand, pressure fluctuations in the clump can reduce the value of $M_{\mathrm{BE}}$ and allow the formation of stars with lower masses. The turnover in the IMF at low masses corresponds to the paucity of cores that form in regions of unusually high pressure and low temperature, where $M_{\mathrm{BE}}$ and therefore $m_{* \min }$ are small. In the Trapezium, the IMF has a broad maximum between 0.6 $M_{\odot}$ and the hydrogen burning limit (Muench et al. 2002). With $\Sigma_{\mathrm{cl}}=0.24 \mathrm{~g} \mathrm{~cm}^{-2}$ from Table $1, \phi_{P}$, core $=1$ (since the low-mass stars are not concentrated in the center of the clump) and $\epsilon_{\text {core }}=0.5$, equations (67) and (72) predict $m_{* \min } \simeq 0.15 M_{\odot}$ in Orion, which is qualitatively consistent with this observation.

\section{PROTOSTELlAR EVOLUTION, LUMINOSITIES AND COMPARISON TO HOT MOLECULAR CORES}

The properties of protostars depend on their accretion rates (e.g., Stahler, Shu \& Taam 1980; Stahler 1988; Palla \& Stahler 1992; Nakano et al. 2000). In particular, the accretion luminosity is

$$
L_{\mathrm{acc}}=\frac{f_{\mathrm{acc}} G m_{*} \dot{m}_{*}}{r_{*}}=4685\left(\frac{f_{\mathrm{acc}}}{0.5}\right)\left(\frac{m_{*}}{30 M_{\odot}} \frac{\dot{m}_{*}}{10^{-4} M_{\odot} \mathrm{yr}^{-1}} \frac{10 R_{\odot}}{r_{*}}\right) L_{\odot},
$$

where $f_{\text {acc }} \leq 1$ is a factor that accounts for the energy advected into the star or used to drive protostellar outflows. If one were able to directly observe the protostar, it would be possible to distinguish the protostellar luminosity from that of the accretion disk. This is generally not possible for massive stars, so here we shall model the luminosities of hot molecular cores (HMCs), ${ }^{4}$ which include the contributions of both the protostar and the accretion disk. The advection of energy into the star at the accretion shock at the stellar surface is usually negligible. However, the mechanical luminosity of the protostellar outflow can reduce $f_{\text {acc }}$ below unity. In Paper II, we show that magneto-centrifugally driven protostellar outflows, which are powered by the rotational energy of the star-disk system, typically extract about half the total available luminosity from gravitational infall. Thus we set $f_{\text {acc }}=0.5$ and note that $L_{\mathrm{acc}} \simeq L_{w}$.

For stars massive enough to be on the main sequence $\left(m_{*} \gtrsim 20 M_{\odot}\right.$ in regions of massive star formation - see below), we can use the zero age main sequence (ZAMS) mass-radius relation to evaluate $r_{*}$. From the solar metallicity models of Schaller et al. (1992), we find $r_{*}=7.27\left(m_{*} / 30 M_{\odot}\right)^{0.55} R_{\odot}$, provided $m_{*}>1.5 M_{\odot}$. The accretion luminosity for stars on the main sequence is then

$$
\begin{aligned}
L_{\mathrm{acc}} & =6360\left(\frac{f_{\mathrm{acc}}}{0.5}\right)\left(\frac{m_{*}}{30 M_{\odot}}\right)^{0.45}\left(\frac{\dot{m}_{*}}{10^{-4} M_{\odot} \mathrm{yr}^{-1}}\right) L_{\odot}, \\
& \rightarrow 3.0 \times 10^{4}\left(\frac{f_{\mathrm{acc}}}{0.5}\right)\left(\frac{m_{* f}}{30 M_{\odot}}\right)^{1.2}\left(\frac{m_{*}}{m_{* f}}\right)^{0.95} \Sigma_{\mathrm{cl}}^{3 / 4} L_{\odot} .
\end{aligned}
$$

The final expression for $L_{\text {acc }}$ (eq.[75]) uses the fiducial massive star accretion rate from equation (41), again for protostars accreting on the ZAMS. For smaller masses the protostar has a size larger than the main sequence size, and the accretion luminosity is correspondingly reduced.

In order to calculate the size of the protostar before it reaches the main sequence one must model protostellar evolution as the star accretes material and burns deuterium. D-burning provides a source of energy to support the star in a configuration with a central temperature $\sim 10^{6} \mathrm{~K}$, so that the star is larger than when on the main sequence (central temperature of $\sim 10^{7} \mathrm{~K}$ ). After a protostar has exhausted its internal store of $\mathrm{D}$, the luminosity available from this process becomes limited by the rate of $\mathrm{D}$ accretion ${ }^{5}$ :

$$
L_{D} \simeq 150\left(\frac{\mathrm{D} / \mathrm{H}}{2.5 \times 10^{-5}}\right)\left(\frac{\dot{m}_{*}}{10^{-4} M_{\odot} \mathrm{yr}^{-1}}\right) L_{\odot} .
$$

Once the star is so massive that this luminosity provides insufficient support, it starts to contract towards the main sequence. This takes of order a Kelvin-Helmholtz time. If the star forms a radiative core during the contraction, shell burning of deuterium temporarily swells the protostellar radius by about a factor of two (Palla \& Stahler 1992).

Most previous investigations of protostellar evolution considered constant accretion rates (e.g. Stahler, Shu \& Taam 1980; Stahler 1988; Palla \& Stahler 1992; Nakano et al. 2000), although Behrend \& Maeder (2001) considered evolution

${ }^{4}$ In our terminology, some observed hot molecular "cores" should properly be termed hot molecular "clumps". The examples chosen here are likely to be true cores.

${ }^{5}$ For consistency with the models of Nakano et al. (1995; 2000) and Palla \& Stahler $(1991 ; 1992)$ we have adopted an interstellar abundance of D relative to $\mathrm{H}$ of $2.5 \times 10^{-5}$ (Bruston et al. 1981; Vidal-Madjar \& Gry 1984). More recent observations tend to favor somewhat smaller abundances in the range $\mathrm{D} / \mathrm{H} \simeq 1.5-2.2 \times 10^{-5}$ (Sonneborn et al. 2000; Moos et al. 2002; Vidal-Madjar \& Ferlet 2002). These results also indicate that there are spatial variations in the $\mathrm{D}$ abundance within the Galaxy. Our adopted value is at the upper end of this observed range. Using a value of $\mathrm{D} / \mathrm{H}=1.6 \times 10^{-5}$ reduces $r_{*}$ by about $30 \%$ for protostellar masses $\gtrsim M_{\odot}$ (see also Stahler 1988). This raises $L_{\text {acc }}$ and thus reduces the estimates of $m_{*}$ listed in Table 3 . However, the effect is very small $(<10 \%)$ for $m_{*} \sim 10 M_{\odot}$, becomes even smaller at higher masses as the internal luminosity becomes more dominant, and disappears completely once the protostar has joined the ZAMS. 
with a growing accretion rate. To consider protostellar evolution with an accretion rate evolving according to equation (66), we have developed a simple model based on that of Nakano et al. (1995; 2000). This model accounts for the total energy of the protostar as it accretes and dissociates matter and, if the central temperature $T_{c} \gtrsim 10^{6} \mathrm{~K}$, burns deuterium. At all times the protostar is assumed to be a polytropic sphere of index, $n$, so that $\gamma_{p}=1+1 / n$. We have modified Nakano et al.'s model to include additional processes, such as deuterium shell burning and the transition between convective and radiative structure, and calibrated these modifications against the more detailed calculations of Stahler (1988) and Palla \& Stahler $(1991 ; 1992)$.

The basic features of our protostellar evolution model, including its differences compared to the Nakano et al. model, are as follows. The initial conditions are set at $m_{*}=0.1 M_{\odot}$ and have a radius that increases with the initial accretion rate (Stahler 1988). Initially the star is contracting and supporting itself by the release of gravitational energy. At this stage convection is imperfect so that $n$ is intermediate between 1.5 and 3 with a weak dependence on $\dot{m}_{*}$, which we calibrate from Palla \& Stahler (1992). Once the star's central temperature reaches that required for D core burning, contraction halts and the star becomes fully convective $(n=1.5)$. We set the $\mathrm{D}$ core burning temperature at $1.5 \times 10^{6} \mathrm{~K}$ (Stahler 1988). While there is sufficient $\mathrm{D}$ to burn, the star maintains this central temperature and its radius grows linearly with mass. After the protostar's D has been exhausted, D burning occurs only at the rate at which it is accreted into the star. Inevitably, as $m_{*}$ grows, the D luminosity (eq. [76]) becomes insufficient to support the star and contraction continues, raising the central temperature.

A radiative barrier forms when the luminosity that can be transported radiatively, $L_{\mathrm{rad}}$, rises to equal $L_{\mathrm{D}}$. In the Nakano et al. model $L_{\mathrm{rad}}$ is approximated by the main-sequence luminosity, $L_{\mathrm{ms}}$, of a star of the same mass. We generalize this and assume that a radiative barrier forms when $L_{D} \leq f_{\mathrm{rad}} L_{\mathrm{ms}}$, where for our adopted zero age main sequence (ZAMS) (Schaller et al. 1992) and by comparison to Palla \& Stahler (1992), $f_{\mathrm{rad}}=0.33$. We approximate the effect of the formation of the radiative barrier by changing the polytropic index of the whole star from $n=1.5$ to 3 . Also at this point D can no longer penetrate to the stellar center so D core burning ceases. However, D will still continue to burn in a shell in regions hotter than $\sim 10^{6} \mathrm{~K}$. We approximate the onset of shell burning to be simultaneous with the formation of a radiative barrier (see Palla \& Stahler 1991 for a more precise description) and we assume it burns at the rate it is accreted. Shell burning swells the stellar radius by approximately a factor of two (Palla \& Stahler 1992), which we take to be a constant in our models, equal to 2.1. In reality the amount of swelling will decrease as $m_{*}$ increases, but we find our simple approximation to be accurate to within $\sim 10 \%$ compared to Palla \& Stahler's results for this stage in the evolution. When $T_{c} \gtrsim 10^{7} \mathrm{~K}$, some hydrogen burning reactions begin and cause the star to become convective once more. The star is rapidly shrinking at this stage, and once its size becomes equal to the ZAMS radius (Schaller et al. 1992), we assume the protostar follows this mass-radius relation for the rest of its growth.

We find our simple one-zone protostellar evolution model matches the mass-radius relations of the $10^{-5}, 3 \times 10^{-5}$ and $10^{-4} M_{\odot}$ constant accretion rate cases with photospheric boundary conditions (no advection) of Palla \& Stahler (1992) to better than about $10 \%$ over the entire evolution to the main sequence. Furthermore this range of accretion rates is similar to that predicted by equation (41) for values of $m_{*}$ relevant to pre-main-sequence evolution and for densities typical of high-mass star-forming clumps. The simplicity of our model allows us to apply it in numerical studies of the formation of clusters of many stars (Tan \& McKee 2002b).

Figure 4 shows the evolution of protostellar radius for $30 M_{\odot}$ stars forming from cores with $k_{\rho}=1.5$ and 1.75 and at pressures equivalent to $\Sigma_{\mathrm{cl}}=1 \mathrm{~g} \mathrm{~cm}^{-2}$. The accretion rate, here and for all cases in this paper, is derived using the two-component core accretion model (equation 66) with $T=100 \mathrm{~K}$. As already discussed, the effect of the thermal core is quite small for the high-pressure conditions considered here. The accretion rate is dominated by gas supported by the nonthermal component. The smaller accretion rate of the $k_{\rho}=1.5$ case leads to $\sim 25 \%$ smaller protostellar radii during most of the evolution compared to the $k_{\rho}=1.75$ case. Figure 5 shows the protostellar radii of stars of different final masses and illustrates the effect of increasing and decreasing the core's surface pressure by factors of 10. From equation (41), the accretion rate increases as $m_{* f}^{3 / 4},\left(m_{*} / m_{* f}\right)^{0.5}$ (for $\left.k_{\rho}=1.5\right)$ and $P_{s}^{3 / 8} \propto \Sigma_{\mathrm{cl}}^{3 / 4}$. Accreting protostars join the main sequence at higher masses for higher accretion rates (Palla \& Stahler 1992). For our fiducial case $\left(k_{\rho}=1.5\right.$ and $\left.\Sigma_{\mathrm{cl}}=1 \mathrm{~g} \mathrm{~cm}^{-2}\right)$, stars join the main sequence at about $20 M_{\odot}$. For larger values of $k_{\rho}$ or $\Sigma_{\mathrm{cl}}$, the accretion rates are higher and stars join the main sequence at somewhat larger masses (e.g., for $m_{* f}=30 M_{\odot}$, a star joins the main sequence at about $24 M_{\odot}$ if either $k_{\rho}=1.75$ or $\left.\Sigma_{\mathrm{cl}} \simeq 3 \mathrm{~g} \mathrm{~cm}^{-2}\right)$. The maximum protostellar radius also increases with accretion rate and thus $\Sigma$ of the clump. Again for $k_{\rho}=1.5,30 M_{\odot}$ stars reach 10-14 $R_{\odot}$ for values of $\Sigma_{\mathrm{cl}}$ within a factor of 3 of those of the typical Plume et al. clumps.

Our model for the evolution of the protostellar radius allows us to make predictions for the evolution of the bolometric luminosity of embedded protostars (Figure 6). The bolometric luminosity is the sum of the internal and accretion luminosities, $L_{\mathrm{bol}}=L_{\mathrm{int}}+L_{\mathrm{acc}}$. We have followed the approximation introduced by Nakano et al. to set the value of $L_{\mathrm{int}}$ approximately equal to $L_{\mathrm{ms}}$, the main sequence luminosity corresponding to the particular value of $m_{*}$, and we evaluate $L_{\mathrm{ms}}$ from the ZAMS models of Schaller et al. (1992). Note that before the protostar joins the ZAMS, the source of $L_{\mathrm{int}}$ is gravitational contraction and $\mathrm{D}$ burning, rather than $\mathrm{H}$ burning. This approximation is least accurate at small stellar masses, but in this regime the total luminosity is dominated by the accretion luminosity. In fact, it should be noted that in the fiducial conditions of massive star forming regions, the turbulent core accretion model predicts quite high accretion luminosities (up to $\sim 10^{3} L_{\odot}$ ) when massive protostars are in their early (low-mass, $\lesssim 3 M_{\odot}$ ) stages of formation.

We compare our theoretical tracks with the observed bolometric luminosities of several HMCs thought to be illuminated internally by massive protostars. For the purpose of this simple analysis we consider three values for the initial column 
density of the clumps in which these stars started forming, $\Sigma_{\mathrm{cl}}=0.32,1.0$ and $3.2 \mathrm{~g} \mathrm{~cm}^{-2}$. This illustrates the effect of a change in the core's surface pressure by a factor of hundred. We also consider values for $k_{\rho}=1.5$ and 1.75 . The observed luminosity and its uncertainty constrain the properties of the protostar. The constraint on $m_{*}$ is shown by the extent of the horizontal error bar for four sources in Figure 6 for the $\Sigma_{\mathrm{cl}}=1 \mathrm{~g} \mathrm{~cm}^{-2}, k_{\rho}=1.5$ case. The full list of constraints (on $m_{*}, m_{* f}$ and $\dot{m}_{*}$ ) and source properties is shown in Table 3 .

If the pressure (or $\Sigma_{\mathrm{cl}}$ ) is lowered, then the stellar mass range implied by a given luminosity moves to higher masses, since the accretion luminosity is reduced and so must be compensated for by an increase in internal stellar luminosity. Similarly, if the pressure is raised, then the stellar mass constraints are lowered. For $L_{\mathrm{bol}} \gtrsim$ few $\times 10^{4} L_{\odot}$ and for the conditions considered here, the internal stellar luminosity (essentially the main-sequence luminosity) tends to dominate that of accretion. In these cases the strong dependence of $L_{\mathrm{ms}}$ on $m_{*}$ means that large pressure uncertainties create only small uncertainties in $m_{*}$.

Osorio et al. (1999) have modeled the infrared and submillimeter spectra of accreting protostars and their surrounding envelopes, and applied their model to the same sources shown in Figure 6 and Table 3. We note that uncertainties in the structure of the gas envelope and the possible contribution from additional surrounding gas cores or diffuse gas will affect the observed spectrum. Comparing results, our inferred stellar masses are similar, except for the lowest luminosity source G34.24+0.13MM; this discrepancy is a result of low value Osorio et al. adopted for the main-sequence luminosity of a $10 M_{\odot}$ star. We systematically favor smaller accretion rates by factors $\sim 2-5$. Their high accretion rates of $\sim 10^{-3} M_{\odot} \mathrm{yr}^{-1}$ for stars with $m_{*} \sim 10 M_{\odot}$ are difficult to achieve unless the pressure is increased substantially; for example, if the stars are all destined to reach $m_{* f} \sim 30 M_{\odot}$, pressure increases of a factor $\sim 40$ are required.

\section{CONCLUSIONS}

We have developed a model (that we term the Turbulent Core Model) for the formation of massive stars, which is an extension of the classic paradigm of low-mass star formation (Shu, Adams, \& Lizano 1987) and is to be contrasted with models involving competitive accretion (Bonnell et al. 1997, 2001) or stellar collisions (Bonnell et al. 1998). The principal motivations for the latter models are the short formation timescales (and correspondingly high accretion rates) mandated by observations of short star cluster formation times (Palla \& Stahler 1999) and theoretical considerations of radiation pressure feedback (Wolfire \& Cassinelli 1987). Such accretion rates are difficult to justify in the standard picture of isothermal core collapse (Shu 1977). Collapse from cores with nonthermal pressure support can involve faster accretion rates (Stahler et al. 1980), but there has been no self-consistent theory for predicting both the normalization of the expected accretion rates and their evolution. This has led to a vast range of massive star accretion rates $\left(10^{-6}-\right.$ $10^{-2} M_{\odot} \mathrm{yr}^{-1}$ ) being considered in the recent literature (e.g. Bernasconi \& Maeder 1996; MP97; Nakano et al. 2000). Conventional theories of massive star formation face further problems: massive stars form preferentially in the centers of stellar clusters (Bonnell \& Davies 1998) where the crowded environment makes it difficult to understand the existence of massive pre-stellar cores, and the high densities and pressures lead to a small thermal Jeans mass that is only a fraction

TABLE 3

Properties of Accreting High-Mass Protostars

\begin{tabular}{|c|c|c|c|c|c|c|}
\hline Source [Ref. $]^{\mathrm{a}}$ & $\begin{array}{c}L_{\mathrm{bol}} \\
\left(10^{3} \mathrm{~L}_{\odot}\right)\end{array}$ & $k_{\rho}$ & $\begin{array}{c}\Sigma_{\mathrm{cl}} \\
\left(\mathrm{g} \mathrm{cm}^{-2}\right)\end{array}$ & $\begin{array}{c}m_{*} \\
\left(M_{\odot}\right)\end{array}$ & $\begin{array}{l}m_{* f} \\
\left(M_{\odot}\right)\end{array}$ & $\begin{array}{c}\dot{m}_{*} \\
\left(10^{-4} \mathrm{M}_{\odot} \mathrm{yr}^{-1}\right)\end{array}$ \\
\hline \multirow[t]{4}{*}{$\mathrm{W} 3\left(\mathrm{H}_{2} \mathrm{O}\right)[1]$} & $10-90$ & 1.5 & 1 & $9.8-23.4$ & $\geq 10.5$ & $2.1-5.6$ \\
\hline & & 1.5 & 0.316 & $10.7-25.1$ & $\geq 10.9$ & $0.9-2.5$ \\
\hline & & 1.5 & 3.16 & $6.2-21.1$ & $\geq 9.5$ & $4.6-12.7$ \\
\hline & & 1.75 & 1 & $7.4-23.4$ & $\geq 10.7$ & $2.1-7.7$ \\
\hline \multirow[t]{4}{*}{ Orion hot core $[2,3]$} & $12.6-50$ & 1.5 & 1 & $10.9-18.1$ & $\geq 11.3$ & $2.2-5.0$ \\
\hline & & 1.5 & 0.316 & $11.5-19.5$ & $\geq 10.7$ & $1.0-2.2$ \\
\hline & & 1.5 & 3.16 & $6.9-17.1$ & $\geq 10.4$ & $4.9-11.4$ \\
\hline & & 1.75 & 1 & $10.5-18.5$ & $\geq 11.6$ & $2.2-7.2$ \\
\hline \multirow[t]{4}{*}{ IRAS $23385+6053[4]$} & $8-32$ & 1.5 & 1 & $8.9-15.3$ & $\geq 9.8$ & $2.0-4.6$ \\
\hline & & 1.5 & 0.316 & $10.0-16.3$ & $\geq 10.1$ & $0.9-2.0$ \\
\hline & & 1.5 & 3.16 & $6.6-14.7$ & $\geq 8.5$ & $4.2-10.5$ \\
\hline & & 1.75 & 1 & $6.6-15.8$ & $\geq 10.0$ & $2.0-6.9$ \\
\hline \multirow[t]{4}{*}{ G34.24+0.13MM [5] } & $1.6-6.3$ & 1.5 & 1 & $3.2-9.0$ & $\geq 4.2$ & $1.1-3.4$ \\
\hline & & 1.5 & 0.316 & $4.3-9.5$ & $\geq 6.0$ & $0.6-1.5$ \\
\hline & & 1.5 & 3.16 & $2.2-7.6$ & $\geq 3.2$ & $2.0-6.3$ \\
\hline & & 1.75 & 1 & $2.6-9.2$ & $\geq 4.3$ & $1.1-5.2$ \\
\hline
\end{tabular}

${ }^{a}$ References: (1) Wyrowski et al. (1999); (2) Kaufman et al. (1998); (3) Wright et al. (1992); (4) Molinari et al. (1998); (5) Hunter et al. (1998). 


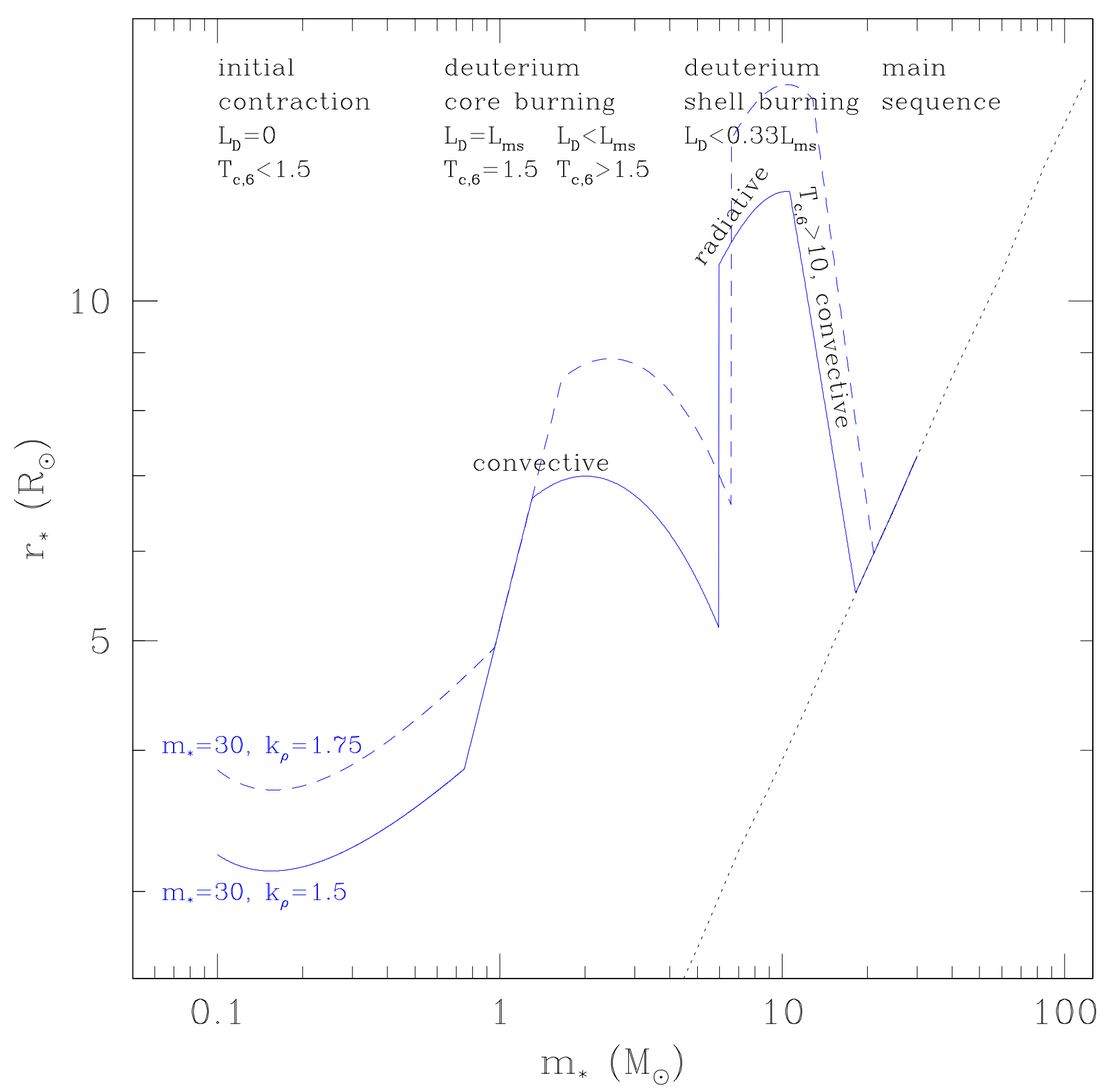

FIG. 4.- Protostellar radius versus $m_{*}$ for stars with $m_{* f}=30 M_{\odot}$ and for $\Sigma_{\mathrm{cl}}=1 \mathrm{~g} \mathrm{~cm}^{-2}$. The solid and dashed lines show the cases with $k_{\rho}=1.5$ and 1.75 , respectively. The dotted line shows the zero age main sequence radius from Schaller et al. (1992). 


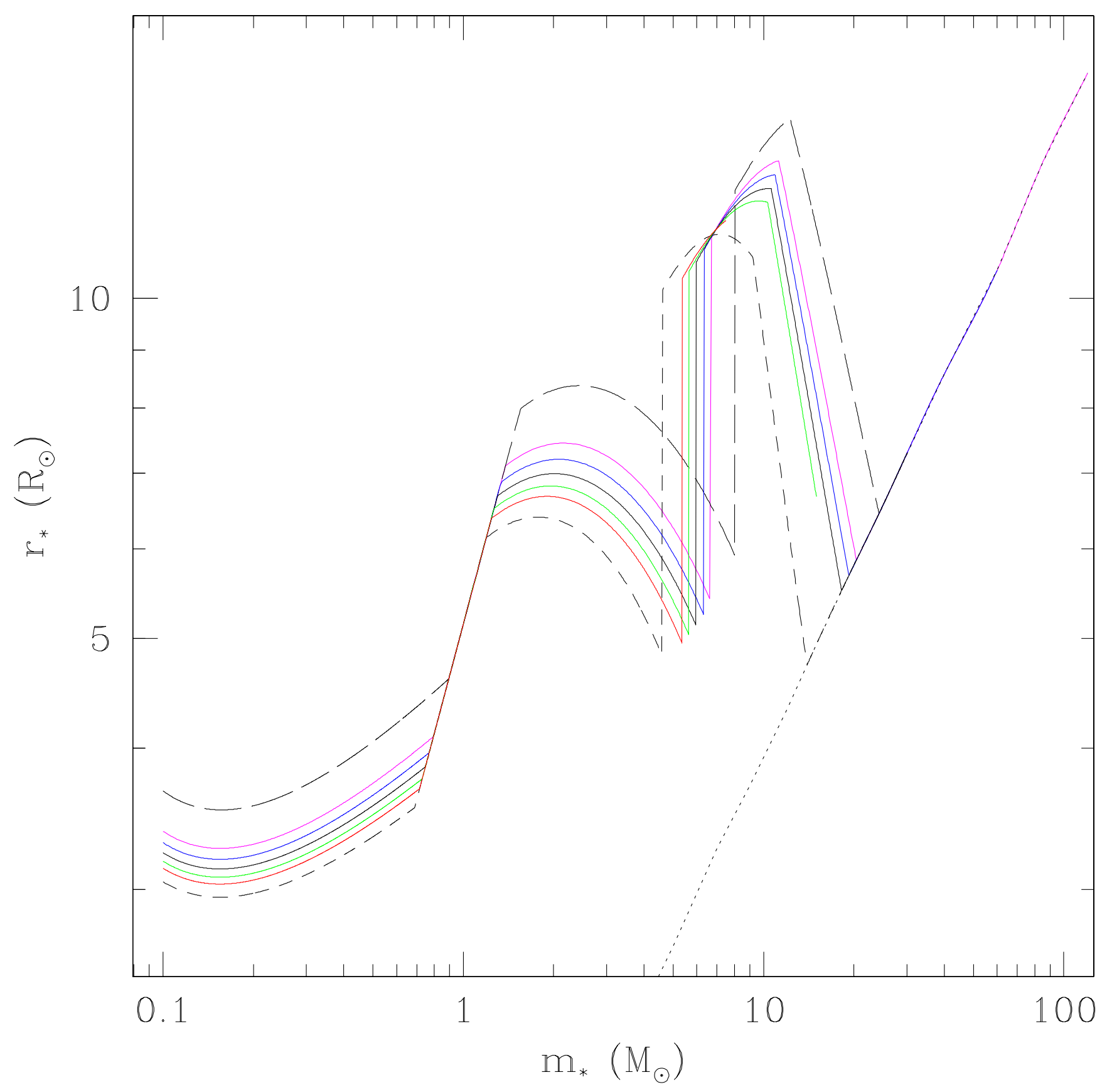

FIG. 5.- Radii of accreting protostars with $k_{\rho}=1.5$. Solid lines show stars of final mass 7.5, 15, 30, 60 and $120 M_{\odot}$ (bottom to top) accreting from cores embedded in a $\Sigma_{\mathrm{cl}}=1 \mathrm{~g} \mathrm{~cm}^{-2}$ clump, typical of Galactic regions observed by Plume et al. (1997). The long dashed and short dashed lines show a $30 M_{\odot}$ star forming in a clump with mean pressure 10 and 0.1 times this value, respectively. The dotted line shows the zero age main sequence radius from Schaller et al. (1992). 


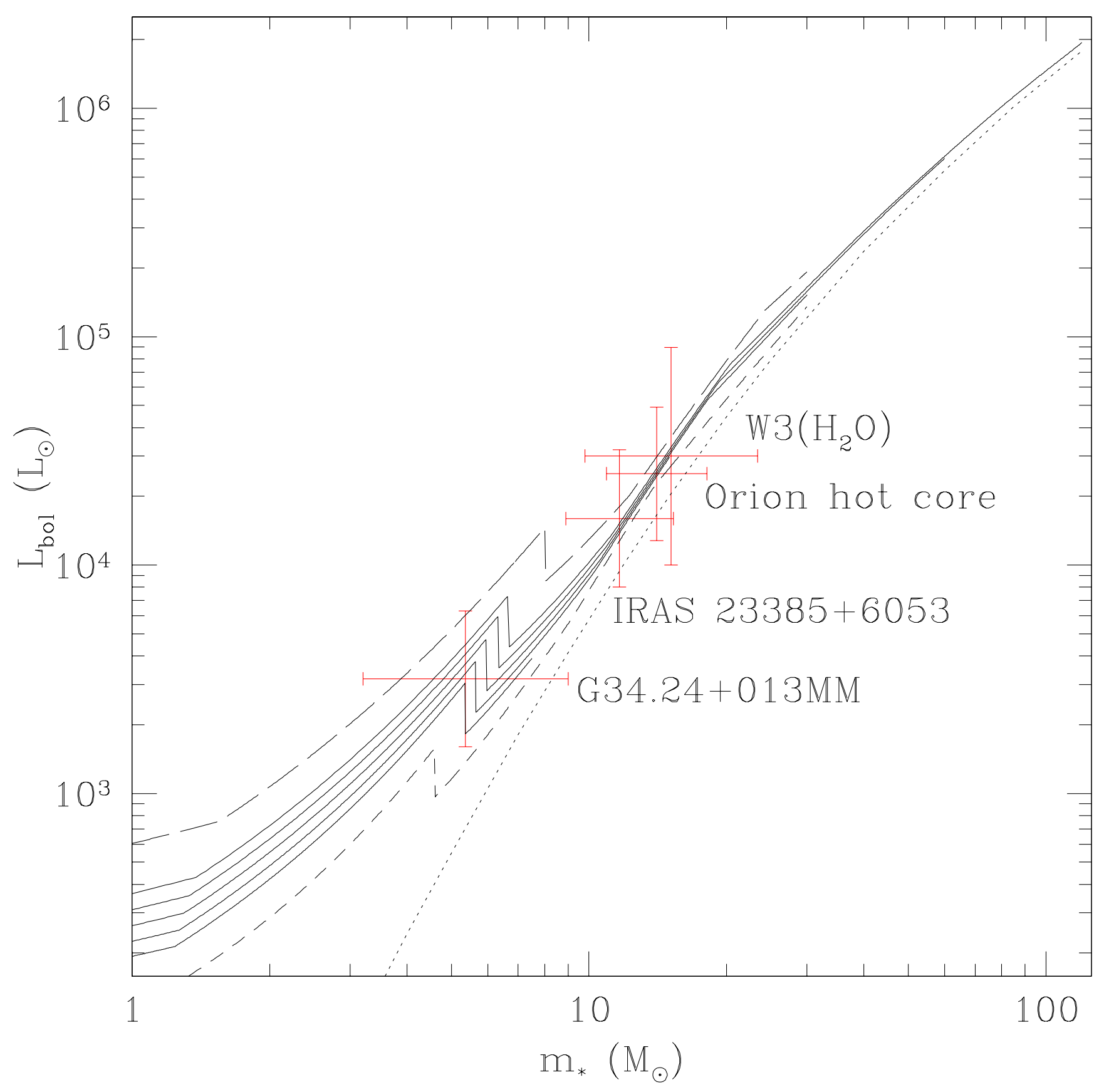

FIG. 6.- Properties of accreting protostars. Solid lines show stars of final mass 7.5, 15, 30, 60 and $120 M_{\odot}$ accreting from cores with $k_{\rho}=1.5$ embedded in a $\Sigma_{\mathrm{cl}}=1 \mathrm{~g} \mathrm{~cm}^{-2}$ clump, typical of Galactic regions observed by Plume et al. (1997). The long dashed and short dashed lines show a $30 M_{\odot}$ star forming in a clump with mean pressure 10 and 0.1 times this value, respectively. The dotted line shows the zero age main sequence luminosity from Schaller et al. (1992). The luminosity step occurring around 5 to $8 M_{\odot}$, depending on the model, corresponds to the onset of deuterium shell burning, which swells the protostellar radius by a factor of about two and thus reduces the accretion luminosity by the same factor. Four observed HMCs are shown. The vertical error bar illustrates the uncertainty in their bolometric luminosities. The horizontal error bar shows the corresponding range of allowed values of $m_{*}$ for the $\Sigma_{\mathrm{cl}}=1 \mathrm{~g} \mathrm{~cm}^{-2}, k_{\rho}=1.5$ models (see Table 3 ). 
of a solar mass (Bonnell et al. 1998).

The above criticisms, taken together with observational hints that massive stars may form differently from low-mass stars (central concentration in star clusters, high degree of equal-mass binarity, complex morphology and extreme energetics of outflows), have motivated collisional and competitive accretion models. However, these are also not without their problems: as noted by Bonnell et al. (1998), competitive (Bondi-Hoyle) accretion is suppressed for stellar masses above $\sim 10 M_{\odot}$ because of radiation pressure feedback, while stellar collisions require extreme $\gtrsim 10^{8} \mathrm{pc}^{-3}$ stellar densities, which have never been observed.

The Turbulent Core Model for massive star formation overcomes the difficulties of the standard accretion scenarios by incorporating the effects of the supersonic turbulence and high pressures observed in massive star-forming regions (Plume et al. 1997). The high pressures mean that cores that become unstable are necessarily very dense and small, leading to high accretion rates and no over-crowding. The turbulent and nonthermal nature of cores gives them substructure and thus the protostar's accretion rate will exhibit fluctuations about the mean. Note that while clumpiness in the core is an attribute of our model for massive star formation, it is not a requirement, as in the collisional model of Stahler et al (2000). The turbulence and nonthermal support of the clump (protocluster) gas determines the IMF for stars above about a solar mass, whereas the Bonnor-Ebert mass is important in determining the IMF at lower masses. While our theory does not aim to predict the IMF, we note that the empirical core mass function is not too different from the stellar one (Testi \& Sargent 1998; Motte et al. 2001), at least up to $\sim 5 M_{\odot}$, the maximum mass probed by these observations. More massive cores, with relatively simple, centrally-concentrated morphologies have been observed, e.g. the Orion hot core (Wright et al. 1992) and $\mathrm{W} 3\left(\mathrm{H}_{2} \mathrm{O}\right)$ (Wyrowski et al. 1999). The stellar IMF would then be set by the core mass function, modulated by $\epsilon_{\text {core }}\left(m_{*}\right)$. In a model in which $\epsilon_{\text {core }}$ is set by the feedback from bipolar outflows, it is found to have a relatively weak dependence on stellar mass (Matzner \& McKee 2000; Paper II).

A natural question is why gravitational fragmentation does not continue down to the thermal Jeans (sub-solar) mass scale inside a massive core, leading to the formation of a cluster of low-mass stars instead of a single massive star. In the self-similar model we have presented, clumps and cores exhibit density fluctuations on all scales down to the thermal Jeans mass. We have assumed that clumps and cores are relatively long-lived (over at least several dynamical times), which implies that most of the density fluctuations existing at a particular instant are gravitationally stable. How good is this assumption? The age spread of pre-main sequence stars in the Orion nebula cluster is of order 1 Myr, which is about an order of magnitude greater than the expected free-fall time of a typical Plume et al. (1997) clump. The approximately spherical morphologies of many clumps observed by Shirley et al. (2002) suggests that they have existed for at least a dynamical timescale. If the onset of gravitational instability is a relatively rare phenomenon in this environment (i.e., $\lesssim$ $20 \%$ of the mass of a clump is undergoing gravitational collapse at any time), as we have assumed, then it is perhaps not surprising that gravitational fragmentation in a collapsing core is unlikely. Gravitational fragmentation is further suppressed by the tidal field of the embedded stars and protostars.

The main results of this paper are the following:

1. The surface densities in observed regions of massive star formation and in star clusters that contain, or did contain, massive stars, are typically within a factor 4 of $\Sigma_{\mathrm{cl}} \sim 1 \mathrm{~g} \mathrm{~cm}^{-2}$. The corresponding mean pressures are $\bar{P} / k \simeq G \Sigma_{\mathrm{cl}}^{2} \sim$ $10^{8}-10^{9} \mathrm{~K} \mathrm{~cm}^{-3}$, much greater than in the diffuse ISM or the typical location in a GMC.

2. Cores have column densities similar to that of the clump in which they are embedded. The mean density of a core significantly exceeds that of its natal clump, and the radius is less than the the tidal radius.

3. Cores that form massive stars are supersonically turbulent; there is no need for the gas to become subsonic in order for star formation to occur.

4. The star-formation time is several times the mean free-fall time of the core out of which the star forms, but is about equal to that of the region in which the core is embedded.

5. The time for a massive star to form in a typical region of of massive star formation is about $10^{5} \mathrm{yr}$, with a weak $\left(m_{* f}^{1 / 4}\right)$ dependence on stellar mass and a somewhat stronger dependence on the surface density of the clump in which it is forming $\left(\Sigma_{\mathrm{cl}}^{-3 / 4}\right)$. This timescale is short compared to estimated cluster formation times, but long compared to the ages of observed supernova remnants, which sometimes have been invoked as star formation triggers.

6. The corresponding accretion rate, approaching $10^{-3} M_{\odot} \mathrm{yr}^{-1}$ for the most massive stars, is high enough to overcome the radiation pressure due to the luminosity of the star.

7. For the typical case we consider, in which the cores out of which the stars form have a density structure $\rho \propto r^{-1.5}$, the protostellar accretion rate grows with time as $\dot{m}_{*} \propto t$. These density structures are consistent with observed clumps and cores, while in an appendix we have shown that logatropic models are inconsistent.

8. The rate at which a core accretes mass from the ambient clump is comparable to the rate at which it processes matter into a star. Once the star has formed, subsequent Bondi-Hoyle accretion is negligible, particularly for massive stars.

9. Presenting a calculation of the evolution of the radius of a protostar, we determine the protostellar accretion luminosity. When the (eventually) massive protostar is still less than a few solar masses, this luminosity can be several hundred to a thousand solar luminosities. Massive protostars join the main sequence at around $20 M_{\odot}$.

10. Application to observations of the Orion hot core suggests a current protostellar mass of between about 11 and $18 M_{\odot}$ and an accretion rate of a few $\times 10^{-4} M_{\odot} \mathrm{yr}^{-1}$. Similar properties are estimated for $\mathrm{W} 3\left(\mathrm{H}_{2} \mathrm{O}\right)$, the Turner-Welch object.

The incorporation of feedback, including protostellar outflows, ionization and radiation pressure, is the subject of Paper 
II. In particular the question of when feedback prevents accretion is addressed. The implications of this work for star cluster formation will be examined in a future paper (see Tan \& McKee 2002b for an initial discussion).

We thank Steve Stahler, Malcolm Walmsley and Richard Larson for helpful discussions. The comments of an anonymous referee improved the clarity of the paper. The research of CFM and JCT has been supported by NSF grant AST-0098365 and by a grant from NASA that supports the Center for Star Formation Studies. JCT has also received support via a Spitzer-Cotsen Fellowship from the Department of Astrophysical Sciences and the Society of Fellows in the Liberal Arts of Princeton University, and from NASA grant NAG5-10811.

\section{APPENDIX}

\section{A. MEAN PRESSURE IN A CLOUD}

We wish to determine the mean pressure in a cloud. We assume that the cloud is ellipsoidal, with radius $R$ normal to the axis of symmetry and radius $Z$ along the axis. The total mass of the cloud is the sum of the gas mass and the stellar mass, $M=M_{\mathrm{g}}+M_{*}$. Recalling that the pressure is related to the effective isothermal sound speed $c$ by $P \equiv \rho c^{2}$, we have

$$
\bar{P} \equiv \frac{1}{V} \int P d V=\bar{\rho}\left\langle c^{2}\right\rangle=\left(\frac{3 M_{\mathrm{g}}}{4 \pi R^{2} Z}\right)\left\langle c^{2}\right\rangle,
$$

where $\bar{\rho}$ is the volume average of the gas density and $\left\langle c^{2}\right\rangle \equiv M_{\mathrm{g}}^{-1} \int c^{2} d M_{\mathrm{g}}$ is the mass average of $c^{2}$.

The virial mass of a spherical cloud is defined by (e.g., Myers \& Goodman 1988)

$$
M_{\text {vir }} \equiv \frac{5\left\langle\sigma^{2}\right\rangle R}{G},
$$

where $\sigma$ is the one-dimensional velocity dispersion. If the pressure in the cloud is entirely due to thermal and turbulent motions of the gas, then $\sigma=c$; if magnetic fields contribute, then $\sigma$ differs from $c$, and we define $\phi_{B} \equiv\left\langle c^{2}\right\rangle /\left\langle\sigma^{2}\right\rangle \geq 1$.

The virial parameter for a spherical cloud is defined as (Bertoldi \& McKee 1992),

$$
\alpha_{\mathrm{vir}} \equiv \frac{5\left\langle\sigma^{2}\right\rangle R}{G M}=\frac{M_{\mathrm{vir}}}{M} .
$$

Note that $\alpha_{\mathrm{vir}}$ is defined in terms of the total mass $M$; the gas mass is a fraction $f_{\mathrm{g}}$ of the total, $M_{\mathrm{g}}=f_{\mathrm{g}} M$.

These definitions are readily extended to non-spherical clouds (Bertoldi \& McKee 1992). For an individual cloud, $R$ is replaced by $R_{\mathrm{obs}}=\left(R_{\mathrm{Max}} R_{\min }\right)^{1 / 2}$, where $R_{\max }$ and $R_{\min }$ are the maximum and minimum observed radii in the plane of the sky. For an ensemble of clouds, the value of $R_{\text {obs }}$ averaged over orientation is denoted by $R_{m}$, and the virial mass and virial parameter are defined in terms of $R_{m}$. In particular, axisymmetric ellipsoidal clouds with a volume $4 \pi R^{2} Z / 3$ have

$$
\phi_{\text {geom }} \equiv \frac{R_{m}^{3}}{R^{2} Z}
$$

in the range $1 \leq \phi_{\text {geom }} \leq 1.41$ for $0.3<Z / R<3$. For both prolate clouds with $Z=2 R$ and oblate clouds with $Z=0.5 R$, we have $\phi_{\text {geom }}=1.13$. Recent observations of star-forming clumps do not show strong evidence for asphericity (Shirley et al. 2002). For our numerical estimates we consider the spherical case with $\phi_{\text {geom }}=1$.

Combining these results, we find that the mean pressure is related to the surface density of the cloud (including any embedded stars), $\Sigma \equiv M / \pi R_{m}^{2}$, by

$$
\bar{P} \equiv\left(\frac{3 \pi f_{\mathrm{g}} \phi_{B} \phi_{\text {geom }} \alpha_{\mathrm{vir}}}{20}\right) G \Sigma^{2}
$$

Note that this is an identity, since it follows directly from the definitions of the various quantities involved. The physics enters primarily in the determination of the virial parameter $\alpha_{\mathrm{vir}}$, which measures the effect of gravity, and in the magnetic parameter $\phi_{B}$. Below, we estimate that $\alpha_{\mathrm{vir}} \simeq 1$ and $\phi_{B} \simeq 2.8$. We define the factor in parentheses in equation (A5) as $\phi_{\bar{P}}$. If we set $f_{\mathrm{g}}=2 / 3$ as in the text and $\phi_{\text {geom }}=1$, then we have

$$
\bar{P} \equiv \phi_{\bar{P}} G \Sigma^{2} \simeq 0.88 G \Sigma^{2} .
$$

\section{A.1. The Virial Parameter $\alpha_{\text {vir }}$}

Existing data suggest that $\alpha_{\mathrm{vir}} \simeq 1$ for gravitationally bound molecular clouds and cores. Solomon et al. (1987) studied GMCs in the first Galactic quadrant and found that the virial masses for the clouds they studied were close to those inferred from gamma ray observations. Such observations determine the $\mathrm{CO}$ to $\mathrm{H}_{2}$ conversion factor, $X \equiv N\left(H_{2}\right) / W_{\mathrm{CO}}$. The most recent determinations of $X$ from EGRET data give $X=1.8 \times 10^{20} \mathrm{~cm}^{-2} / \mathrm{K} \mathrm{km} \mathrm{s}^{-1}$ in the local ISM (Dame, Hartmann, \& Thaddeus 2001) and $X=1.56 \times 10^{20} \mathrm{~cm}^{-2} / \mathrm{K} \mathrm{km} \mathrm{s}^{-1}$ in the Galactic plane (Hunter et al. 1997). Determining $X$ from virial masses, Solomon et al. found $X_{\text {vir }}=2.2 \times 10^{20} / M_{6}^{0.235} \mathrm{~cm}^{-2} / \mathrm{K} \mathrm{km} \mathrm{s}^{-1}$, where $M_{6}$ is the cloud mass in units of $10^{6} M_{\odot}$. [Their masses have been increased by a factor 1.31 to allow for a reduction in the distance to the Galactic Center from 10 to $8.5 \mathrm{kpc}$ (a factor 0.85), for an increase in the mass due to the extrapolation to zero intensity recommended by Solomon and Rivolo (1989; a factor 1.4), and a difference in the definition of $M_{\mathrm{vir}}$ (a factor 1.1).] Note that $X_{\mathrm{vir}} / X=N_{\mathrm{vir}} / N=M_{\mathrm{vir}} / M=\alpha_{\mathrm{vir}}$. For a typical cloud mass of $10^{6} M_{\odot}$ in the Galactic plane, this gives 
$\alpha_{\mathrm{vir}}=X_{\mathrm{vir}} / X($ Galactic plane $)=1.4$. Note that the gamma-ray determination of $X$ is necessarily biased toward nearby clouds, which have an average mass somewhat less than $10^{6} M_{\odot}$, which could raise $\alpha_{\text {vir }}$ somewhat. In any case, Solomon et al. estimated their cloud masses based on the assumption that $\alpha_{\mathrm{vir}}=1.1$; if it is in fact larger than this, then their masses should be reduced by a factor $1.1 / \alpha_{\text {vir }}$. Dame et al. (1986) surveyed the brightest CO clouds in the first Galactic quadrant and determined cloud masses with $X=1.6 \times 10^{20} \mathrm{~cm}^{-2} / \mathrm{K} \mathrm{km} \mathrm{s}^{-1}$. Reducing their masses by $0.85^{2}$ and their radii by 0.85 to adjust to our adopted distance to the Galactic Center, we find that the mean virial parameter in their cloud sample is $\alpha_{\mathrm{vir}}=1.3$, with a standard deviation of 0.76 . Their results do not give a dependence of $\alpha_{\mathrm{vir}}$ on cloud mass as found by Solomon et al. (1987), although it must be kept in mind that the Dame et al. sample is considerably smaller.

Both bound and unbound structures exist within GMCs. Bertoldi \& McKee (1992) found that most ${ }^{13}$ CO clumps are gravitationally unbound; however, the most massive ones, which were also the ones that showed evidence for star formation, appeared bound. Tachihara, Mizuno, \& Fukui (2000) have studied the $\mathrm{C}^{18} \mathrm{O}$ cores in Ophiuchus and shown that they are generally bound. In the northern region, the value of $\alpha_{\text {vir }}$ calculated from the mean properties of the clouds (and including the thermal contribution of $\mathrm{H}$ and $\mathrm{He}$ to $\sigma^{2}$ ) is 2.2 (excluding one core with $M_{\mathrm{vir}}=12 M$ ), whereas in the main body of Ophiuchus it is 0.9. In Taurus, Onishi et al. (1996) found $40 \mathrm{C}^{18} \mathrm{O}$ cores with $\alpha_{\text {vir }}=0.9$ as calculated from the mean properties (and with the same correction to $\alpha_{\mathrm{vir}}$ ). The fraction of these cores that have embedded young stellar objects is $16 \%, 33 \%$, and $48 \%$ in the northern region, main body of $\rho$ Oph, and Taurus, respectively. The two regions with the most active star formation thus have $\alpha_{\mathrm{vir}} \sim 1$. However, these data refer only to regions of low-mass star formation. There are no data from which one can directly determine the virial parameter for massive-star forming regions such as those studied by Plume et al. (1997).

The value of $\alpha_{\text {vir }}$ can also be estimated from theory. Let $\mathcal{T}=\frac{3}{2} M\left\langle\sigma^{2}\right\rangle$ be the total kinetic energy (including thermal energy) and $\mathcal{W} \equiv \frac{3}{5} a G M^{2} / R$ be the gravitational energy; the parameter $a$ is the ratio of the gravitational energy to that of a uniform sphere. The virial parameter is then $\alpha_{\text {vir }}=2 a \mathcal{T} /|\mathcal{W}|$. Including the effects of the surface pressure $P_{s}$ and the magnetic fields, the virial theorem implies

$$
\alpha_{\mathrm{vir}}=\frac{a}{\left(1-P_{s} / \bar{P}\right)}\left(1-\frac{\mathcal{M}}{|\mathcal{W}|}\right),
$$

where $\mathcal{M}$ is the magnetic energy (Bertoldi \& McKee 1992). The value of $\mathcal{M} /|\mathcal{W}|$ is quite uncertain, however.

In the text, we model the cores as singular polytropic spheres (SPSs). If we adopt the same model for the clump in which the cores are embedded, we have

$$
\alpha_{\mathrm{vir}}=\frac{\left\langle\sigma^{2}\right\rangle}{\left\langle c^{2}\right\rangle}\left(\frac{5\left\langle c^{2}\right\rangle R}{G M}\right)=\frac{\alpha_{\mathrm{SPS}}}{\phi_{B}},
$$

where

$$
\alpha_{\mathrm{SPS}}=\frac{5}{2}\left(\frac{4-3 \gamma_{p}}{6-5 \gamma_{p}}\right)\left(\frac{2-\gamma_{p}}{\gamma_{p}}\right)
$$

is the virial parameter of an SPS (see MP96 or McKee \& Holliman 1999). As discussed in $\S 3$, observed clumps have density distributions characterized by values of $k_{\rho}$ ranging from 1 to 2 , with 1.5 being typical. The value of $\gamma_{p}$ corresponding to $k_{\rho}=1.5$ is $2 / 3$, which gives $\alpha_{\mathrm{SPS}}=15 / 4$. Below, we estimate $\phi_{B}=2.8$ so that $\alpha_{\mathrm{vir}}=1.34$, which is comparable to the observed values.

\section{A.2. The Magnetic Parameter $\phi_{B}$}

The effective sound speed $c^{2} \equiv P / \rho$ includes the contributions of both the gas pressure and the magnetic pressure,

$$
c^{2}=\sigma^{2}+\frac{B^{2}}{8 \pi \rho}+\frac{\delta B^{2}}{24 \pi \rho},
$$

where $\sigma$ is the 1-D velocity dispersion, which can be inferred from observation. We have distinguished between the pressure associated with the background field $B$ (pressure $=$ energy density) and that associated with a turbulent field $\delta B$ [assumed to have a random orientation so that pressure $=(1 / 3)$ energy density]. Taking the mass average of this relation gives

$$
\left\langle c^{2}\right\rangle=\left\langle\sigma^{2}\right\rangle\left(1+\frac{3}{2} \frac{E_{B}}{E_{K}}+\frac{1}{2} \frac{E_{\delta B}}{E_{K}}\right),
$$

where $E_{B}=\int\left(B^{2} / 8 \pi\right) d V$ is the background field energy, $E_{K}=\frac{3}{2} f_{\mathrm{g}} M\left\langle\sigma^{2}\right\rangle$ is the total kinetic energy of the gas, and $E_{\delta B}$ is the energy of the turbulent field. The second term can be rewritten in terms of an average Alfven Mach number $m_{A}$, where

$$
m_{A}^{2} \equiv \frac{3\left\langle\sigma^{2}\right\rangle}{\left\langle v_{A}^{2}\right\rangle}=\frac{E_{K}}{E_{B}}
$$

We now show that the last term in equation (A11) is about $1 / 3$. Fluctuations in the magnetic field are associated with motions perpendicular to the field, and, for small amplitudes and negligible ambipolar diffusion, these motions are in equipartition with the fluctuating field (Zweibel \& McKee 1995): (1/2) $\rho v_{\perp}^{2}=\delta B^{2} / 8 \pi$. There are also motions along 
the field; assuming that the velocity field is approximately isotropic $\left(v_{\perp}^{2}=2 \sigma^{2}\right)$, we find $(2 / 3) E_{K}=E_{\delta B}$. Thus, for isotropic turbulence the last term is $1 / 3$. One can show that the same result is obtained if one considers the fluctuations to be MHD waves in a cold plasma and evaluates the wave pressure using the results of Dewar (1970). This result is in reasonably good agreement with the simulations of supersonic MHD turbulence by Stone, Ostriker, and Gammie (1998), who found $E_{\delta B} / 2 E_{K}=(0.28,0.30)$ for $\beta \equiv 8 \pi P_{\mathrm{th}} / B^{2}=(0.01,0.1)$, respectively. We therefore adopt $E_{\delta B} / 2 E_{K}=0.3$ for low $\beta$, supersonic turbulence, which is the type that appears to be characteristic in molecular clouds. The effect of the magnetic field is then described by

$$
\phi_{B} \equiv \frac{\left\langle c^{2}\right\rangle}{\left\langle\sigma^{2}\right\rangle}=1+\frac{3}{2} \frac{E_{B}}{E_{K}}+\frac{E_{\delta B}}{2 E_{K}}=1.3+\frac{3}{2 m_{A}^{2}}
$$

from equation (A11). This equation does not apply to high- $\beta$ plasmas, which have $\phi_{B} \simeq 1$.

The magnitude of the magnetic field in massive-star forming clumps is unknown. The most complete survey of magnetic field measurements in star-forming regions is that of Crutcher (1999). While this survey primarily deals with regions of low-mass star formation, it does include regions whose density is comparable to, and even greater than, that in the Plume et al. clumps. For regions in which the magnetic field could be measured, the median value of the Alfven Mach number is 1.0. The magnetic field factor is then $\phi_{B}=1.3+1.5=2.8$.

\section{B. THE FAILURE OF LOGATROPES}

Turbulent regions have velocity dispersions that increase with scale. Larson (1981) found that molecular clouds have line widths that increase with size at a rate intermediate between that of Kolmogorov turbulence $\left(\left\langle\sigma^{2}\right\rangle^{1 / 2} \propto r^{1 / 3}\right)$ and that of Burgers' turbulence, which is appropriate for a system of shocks $\left(\left\langle\sigma^{2}\right\rangle^{1 / 2} \propto r^{1 / 2}\right)$. Subsequent observations have shown that GMCs (Solomon et al 1987; Heyer \& Schloerb 1997) and low-mass cores (e.g., Caselli \& Myers 1995) have line widths that increase approximately as $r^{1 / 2}$. Larson (1981) also found that the density tends to fall off as $1 / r\left(\right.$ i.e., $k_{\rho}=1$ ), so that in these regions there is a relation between the velocity dispersion and density, $\left\langle\sigma^{2}\right\rangle^{1 / 2} \propto \rho^{-1 / 2}$. As discussed in the text, regions of high-mass star formation typically have $k_{\rho} \simeq 1.5$, so they usually do not satisfy this line-width density relation. Nonetheless, inferences drawn about the properties of turbulence in low-mass cores and GMCs would have implications for high-mass cores as well.

Before continuing, we note that Larson's relations primarily refer to properties of an ensemble of clouds, rather than to the structure of individual clouds. The relation $n \propto 1 / r$ then means that the column densities of different clouds are all about the same, to within an order of magnitude. (This can be understood in terms of the conditions needed for the stability of molecular clouds-see Elmegreen 1989; McKee 1999.) If this relation applied within individual clouds, and if the line-width size relation $\left\langle\sigma^{2}\right\rangle^{1 / 2} \propto r^{1 / 2}$ also applied, then the turbulent pressure $\rho\left\langle\sigma^{2}\right\rangle$ would be constant and would not exert a force. It is for this reason that the density that can be supported by a self-similar cloud in hydrostatic equilibrium (eq. 4) vanishes for $k_{\rho}=1$.

Lizano \& Shu (1989) pointed out that the line-width density relation $\left\langle\sigma^{2}\right\rangle^{1 / 2} \propto \rho^{-1 / 2}$ follows if one assumes that (1) the turbulent pressure is proportional to the logarithm of the density, $P_{\text {turb }} \propto \ln \rho$, since then the signal speed $(d P / d \rho)^{1 / 2}$ varies as $\rho^{-1 / 2}$, and $(2)$ the velocity dispersion $\sigma$ varies as the signal speed $(d P / d \rho)^{1 / 2}$. Including the thermal pressure of an isothermal gas, they wrote the total pressure as

$$
P=\rho c_{\mathrm{th}}^{2}+K \ln \left(\rho / \rho_{0}\right)
$$

where $K$ is a numerical constant and $\rho_{0}$ is a reference density. Gehman et al. (1996) termed this a "logatropic" equation of state. Note that logatropes are not self-similar, and therefore avoid the "no-force" problem that afflicts self-similar polytropes with $k_{\rho}=1$.

MP96 pointed out a fundamental problem with this formulation: Since the central velocity dispersion is observed to be thermal, it is necessary to set $\rho_{0}=\rho_{c}$, which Gehman et al. (1996) did. However, if one then sets $K \gg 1$, as both Lizano \& Shu (1989) and Gehman et al. (1996) did, then the pressure becomes negative when the density drops only slightly from its central value. For example, if $K=10$, the pressure is already negative for $\rho=0.9 \rho_{c}$.

To overcome this problem, MP96 proposed an alternate form of the logatropic equation of state,

$$
P=P_{c}\left[1+A_{\mathrm{MP}} \ln \left(\rho / \rho_{c}\right)\right],
$$

where $A_{\mathrm{MP}}$ is a numerical constant that they estimate is about 0.18 in observed molecular clouds. (We have added a subscript "MP" to the parameter $A$ defined by McLaughlin \& Pudritz in order to distinguish it from the parameter defined in the text. We emphasize that our model in the main text is based on the polytropic results of McLaughlin \& Pudritz, not the logatropic ones.) They present a thorough study of the structure and stability of spherical clouds with this equation of state. In particular they show that for $A_{\mathrm{MP}} \ll 1$ and at large $r$, the density approaches the form

$$
\rho=\rho_{c}\left(r_{1} / r\right),
$$

where

$$
r_{1} \equiv\left(\frac{A_{\mathrm{MP}} \sigma_{c}^{2}}{2 \pi G \rho_{c}}\right)^{1 / 2}
$$

indeed, the density is quite close to this value for $r \gtrsim 1.5 r_{1}$. Gravitationally bound clouds have radii $R \gg r_{1}$. MP96 focussed on clouds near the brink of instability, which have $R / r_{1}=\exp \left(1 / A_{\mathrm{MP}}-1 / 4\right) \rightarrow 200$, where the numerical 
evaluation is for $A_{\mathrm{MP}}=0.18$. The collapse of logatropic spheres was studied analytically by MP97 and numerically by Reid, Pudritz, \& Wadsley (2002).

Unfortunately, this form of the logatropic equation of state is also fundamentally flawed: It satisfies the observed linewidth size relation only when the cloud is gravitationally unbound; furthermore, it leads to a "droop" in the line-width size relation for clouds that are bound. To see this, we evaluate the properties of the MP-logatrope in the case $r \gg r_{1}$, so that $\rho \propto 1 / r:$

$$
\begin{aligned}
M & =2 \pi \rho_{c} r_{1} R^{2}, \\
\frac{\left\langle\sigma^{2}\right\rangle}{\sigma_{c}^{2}} & =\frac{1}{M \sigma_{c}^{2}} \int P d V=\frac{2 R}{3 r_{1}}\left[1+\frac{1}{3} A_{\mathrm{MP}}-A_{\mathrm{MP}} \ln \left(\frac{R}{r_{1}}\right)\right], \\
\alpha_{\mathrm{vir}} & \equiv \frac{5\left\langle\sigma^{2}\right\rangle R}{G M}=\left(\frac{10}{3 A}\right)\left[1+\frac{1}{3} A_{\mathrm{MP}}-A_{\mathrm{MP}} \ln \left(\frac{R}{r_{1}}\right)\right],
\end{aligned}
$$

where the virial parameter $\alpha_{\mathrm{vir}}$ is discussed in $\S$ A.1. Since the effects of magnetic fields have not been taken into account here, this value of the virial parameter corresponds to $\alpha_{\text {non }}$ in the notation of MP96. If magnetic fields are important, then MP96 assume that $\alpha_{\text {non }}$ is still given by the rightmost expression in equation (B7), while the observed value (which they denote $\alpha_{\mathrm{mag}}$ ) is reduced. In terms of the parameter $\phi_{B}$ introduced in Appendix A, we have $\alpha_{\mathrm{mag}}=\alpha_{\mathrm{non}} / \phi_{B}$.

Evaluating the exponent in the line-width size relation for MP-logatropes, we find

$$
\frac{d \ln \left\langle\sigma^{2}\right\rangle}{d \ln r}=1-\frac{10}{3 \alpha_{\text {vir }}} .
$$

This result immediately reveals the two flaws described above. First, these logatropes obey the observed line-width size relation $\left\langle\sigma^{2}\right\rangle \propto r$ only when the virial parameter $\alpha_{\text {vir }} \gg 10 / 3$, corresponding to an observed virial parameter $\alpha_{\text {mag }} \gg 1$; i.e., the clouds are unbound. Second, when clouds are strongly bound (i.e., nearly critical), the line width actually decreases with size. This effect is clearly apparent in Figure 2 of MP96. In particular, for a critical cloud, we have $\alpha_{\text {vir }}=35 / 18$ (eq. B7; MP96) so that the slope of the line-width size relation is $-5 / 7$. Such a "droop" in the line-width size relation has never been observed. For example, the observations of four GMCs by Heyer \& Schloerb (1997) show that in each case the velocity dispersion rises approximately as $r^{1 / 2}$ over the entire range of their observations. (However, it should be noted that observations of high-mass star-forming clumps are not yet sufficiently detailed to rule out the possibility of a droop in the line-width size relation in these objects.) We conclude that, where tested by observation, this form of the logatropic equation of state also fails.

\section{REFERENCES}

Adams, F. C., \& Fatuzzo, M. 1996, ApJ, 464, 256

Andre, P., Ward-Thompson, D., and Barsony, M. 2000, in Protostars 8 Planets $I V$, eds. V. Mannings, A. P. Boss, \& S. S. Russell (Tucson: The University of Arizona Press), p. 59

Basu, S. 2000, ApJ, 540, L103

Behrend, R., \& Maeder, A. 2001, A\&A, 373, 190

Bernasconi, P. A., \& Maeder, A. 1996, A\&A, 307, 829

Bertoldi, F., \& McKee, C. F. 1992, ApJ, 395, 140

Beuther, H., Schilke, P., Menten, K. M., Motte, F., Sridharan, T. K., \& Wyrowski, F. 2002, ApJ, 566, 945

Binney, J., \& Merrifield, M. 1998, Galactic Astronomy (Princeton University Press)

Bondi, H., \& Hoyle, F. 1944, MNRAS, 104, 273

Bonnell, I. A., Bate, M. R., Clarke, C. J., \& Pringle, J. E. 1997, MNRAS, 285, 201

Bonnell, I. A., Bate, M. R., \& Zinnecker, H. 1998, MNRAS, 298, 93

Bonnell, I. A., \& Davies, M.B. 1998, MNRAS, 295, 691

Bonnell, I. A., Clarke, C. J., Bate, M. R., \& Pringle, J. E. 2001, MNRAS, 324, 573

Bonnor, W.B. 1956, MNRAS, 116, 350

Bruston, P., Audouze, J., Vidal-Madjar, A., \& Laurent, C. 1981, ApJ, 243, 161

Caselli, P., \& Myers, P. C. 1995, ApJ, 446, 665

Crutcher, R. M. 1999, ApJ, 520, 706

Curry, C. L., \& McKee, C. F. 2000, ApJ, 528, 734

Dame, T.M., Elmegreen, B.G., Cohen, R.S., \& Thaddeus, P. 1986, ApJ, 305, 892

Dame, T.M., Hartmann, D., and Thaddeus, P. 2001, ApJ, 547, 792

DeMarchi et al. 1997, ApJ, 479, L27

Dewar, R.L. 1970, Phys. Fluids, 13, 2710

Doty, S. D., van Dishoeck, E. F., van der Tak, F. F. S., \& Boonman, A. M. S. 2002, A\&A, accepted, (astro-ph 0205292)

Ebert, R. 1955, Z. Astrophys., 37, 216

Elmegreen, B. G. 1989, ApJ, 338, 178

Elmegreen, B. G., \& Efremov, Y. N. 1997, ApJ, 480, 235

Elmegreen, B. G., \& Falgarone, E. 1996, ApJ, 471, 816

Evans, N. J., II, Shirley, Y. L., Mueller, K. E., \& Knez, C. 2002, in Hot Stars Workshop III: The Earliest Phases of Massive Star Birth, ed. P. A. Crowther (ASP)
Figer, D. F., Kim, S. S., Morris, M., Serabyn, E., Rich, R. M., \& McLean, I. S. 1999, ApJ, 525, 750

Gehman, C.S., Adams, F.C., Fatuzzo, M., \& Watkins, R. 1996, ApJ, 457,718

Gilbert, A.M., \& Graham, J.R. 2001, AAS Meeting 199, \#14.04

Goodman, A.A., Benson, P.J., Fuller, G.A., \& Myers, P.C. 1992, ApJ, 406, 528

Henriksen, R. N., \& Turner, B. E. 1984, ApJ, 287, 200

Henriksen, R. N. 1986, ApJ, 310, 189

Heyer, M.H., \& Schloerb, F.P. 1997, ApJ, 475, 173

Hillenbrand, L.A., \& Hartmann, L.W. 1998, ApJ, 492, 540

Hills, J. G. 1980, ApJ, 225, 986

Ho, L., \& Filippenko, A. 1996, ApJ, 466, L83

Hunter, S. D., et al. 1997, ApJ, 481, 205

Hunter, T. R., Neugebauer, G., Benford, D. J., Matthews, K., Lis, D. C., Serabyn, E., \& Phillips, T. G. 1998, ApJ, 493, L97

Jijina, J., \& Adams, F. C. 1996, ApJ, 462, 874

Jijina, J., Myers, P.C., \& Adams, F. C. 1999, ApJS, 125, 161

Kahn, F. D. 1974, A\&A, 37, 149

Kaufman, M. J., Hollenbach, D. J., \& Tielens, A. G. G. M. 1998, ApJ, 497, 276

Kim, S. S., Figer, D. F., Lee, H. M., \& Morris, M. 2000, ApJ, 545, 301

Lada, C.J. 1999, in The Origin of Stars and Planetary Systems, ed. C.J. Lada \& N.D. Kylafis (Dordrecht: Kluwer), 143

Larson, R. B. 1981, MNRAS, 194, 809

Larson, R. B., \& Starrfield, S. 1971, A\&A, 13, 190

Li, Z.-Y., \& Shu, F. H. 1997, ApJ, 475, 237

Lizano, S., \& Shu, F.H. 1989, ApJ, 342, 834

Maloney, P. 1988, ApJ, 334, 761

Matzner, C. D., \& McKee, C. F. 2000, ApJ, 545, 364

McKee, C. F. 1999, in The Origin of Stars and Planetary Systems, ed. C.J. Lada \& N.D. Kylafis (Dordrecht: Kluwer), 29

McKee, C. F., \& Holliman, J. H. 1999, ApJ, 522, 313

McKee, C. F., \& Tan, J. C. 2002, Nature, 416, 59 (MT)

McKee, C. F., \& Zweibel, E. G. 1995, ApJ, 440, 686

McLaughlin, D. E., \& Pudritz, R. E. 1996, ApJ, 469, 194 (MP96)

McLaughlin, D. E., \& Pudritz, R. E. 1997, ApJ, 476, 750 (MP97) 
Molinari, S., Testi, L., Brand, J., Cesaroni, R., \& Palla, F. 1998, ApJ, 505, L39

Moos, H. W., et al. 2002, ApJS, 140, 3

Motte, F., André, P., Ward-Thompson, D., \& Bontemps, S. 2001, A\&A, 372, L41

Mueller, K. E., Shirley, Y. L., Evans, N. J., II, \& Jacobson, H. R. 2002a, in Hot Stars Workshop III: The Earliest Phases of Massive Star Birth, ed. P. A. Crowther (ASP)

Mueller, K. E., Shirley, Y. L., Evans, N. J., II, \& Jacobson, H. R. 2002b, ApJS, accepted (astro-ph/0207322)

Muench, A. A., Lada, E. A., Lada, C. J., \& Alves, J. 2002, ApJ, 573, 366

Myers, P. C., \& Fuller, G. A. 1992, ApJ, 396, 631

Myers, P. C., \& Goodman, A. A. 1988, ApJ, 329, 392

Myers, P. C., Fuller, G. A., Goodman, A. A., \& Benson, P.J. 1991, ApJ, 376, 561

Nakano, T., Hasegawa, T., \& Norman, C. 1995, ApJ, 450, 183

Nakano, T., Hasegawa, T., Morino, J.-I., \& Yamashita, T. 2000, ApJ, 534,976

Onishi, T., Mizuno, A., Kawamura, A., Ogawa, H., \& Fukui, Y. 1996, ApJ, 465, 815

Ossenkopf, V., \& Henning, Th. 1994, A\&A, 291, 943

Osorio, M., Lizano, S., \& D'Alessio, P. 1999, ApJ, 525, 808

Paczynski, B. 1971, ARAA 9, 183

Palla, F., \& Stahler, S. W. 1991, ApJ, 375, 288

Palla, F., \& Stahler, S. W. 1992, ApJ, 392, 667

Palla, F., \& Stahler, S. W. 1999, ApJ, 525, 722

Patel, K., \& Pudritz, R. E. 1994, ApJ, 424, 688

Plume, R., Jaffe, D. T., Evans, N. J., II, Martin-Pintado, J., \& Gomez-Gonzalez, J. 1997, ApJ, 476, 730

Pollack, J.B., Hollenbach, D., Beckwith, S., Simonelli, D.P., Roush, T., \& Fong, W. 1994, ApJ, 421, 615

Reid, M. A., Pudritz, R. E., \& Wadsley, J. 2002, ApJ, 570, 231

Richer, J., Shepherd, D. Cabrit, S., Bachiller, R., \& Churchwell, E. 2000, in Protostars \&s Planets IV, eds. V. Mannings, A. P. Boss, \& S. S. Russell (Tucson: The University of Arizona Press), 867

Schaerer, D., \& de Koter, A. 1997, A\&A, 322, 598

Schaller, G., Schaerer, D., Meynet, G., \& Maeder, A. 1992, A\&AS, 96, 269

Shirley, Y. L., Evans II, N. J., Mueller, K. E., Knez, C., \& Jaffe, D. T. 2002, ApJS, submitted.

Shu, F. H. 1977, ApJ, 214, 488
Shu, F. H., Adams, F. C., \& Lizano, S. 1987, ARA\&A, 25, 23

Solomon, P.M, \& Rivolo, A.R. 1989, ApJ, 339, 919

Solomon, P.M., Rivolo, A.R., Barrett, J., \& Yahil, A. 1987, ApJ, 319, 730

Sonneborn, G., Tripp, T. M., Ferlet, R., Jenkins, E. B., Sofia, U. J. Vidal-Madjar, A., \& Wozniak, P. R. 2000, ApJ, 545, 277

Spitzer, L. 1987, Dynamical Evolution of Globular Clusters (Princeton University Press)

Stahler, S. W. 1988, ApJ, 332, 804

Stahler, S. W., Shu, F. H., \& Taam, R. E. 1980, ApJ, 241, 637

Stahler, S. W., Palla, F., \& Ho, P. T. P. 2000, in Protostars $\& 3$ Planets $I V$, eds. V. Mannings, A. P. Boss, \& S. S. Russell (Tucson: The University of Arizona Press), 327

Stone, J.M., Ostriker, E.G., \& Gammie, C.F. 1998, ApJ, 508, L99

Tachihara, K., Mizuno, A., \& Fukui, Y. 2000, ApJ, 528, 817

Tan, J. C., \& McKee, C. F. 2002a, in preparation (Paper II)

Tan, J. C., \& McKee, C. F. 2002b, in Hot Stars Workshop III: The Earliest Phases of Massive Star Birth, ed. P. A. Crowther (ASP)

Testi, L., \& Sargent, A. I. 1998, ApJ, 508, L91

Turner, J. L., Beck, S. C., \& Ho, P. T. P. 2000, ApJ, 532, L109

van den Bergh, S., Morbey, C., \& Pazder, J. 1991, ApJ, 375, 594

van der Tak, F. F. S., van Dishoeck, E. F., Evans, N. J., II, \& Blake, G. A. 2000, ApJ, 537, 283

Vazquez-Semadeni, E., Ballesteros-Paredes, J., \& Klessen, R. 2002, in Star Formation Across the Mass Spectrum, ed. J. de Buizer (San Francisco: ASP), in press

Vazquez-Semadeni, E., Ostriker, E., Passot, T., Gammie, C.F., \& Stone, J.M. 2000, in Protostars \& Planets IV, eds. V. Mannings, A. P. Boss, \& S. S. Russell (Tucson: The University of Arizona Press), 3

Vidal-Madjar, A., \& Ferlet, R. 2002, ApJ, 571, L169

Vidal-Madjar, A., \& Gry, C. 1984, A\&A, 138, 285

Williams, J. P., \& McKee, C. F. 1997, ApJ, 476, 166

Williams, J. P., Blitz, L., \& McKee, C.F. 2000, in Protostars \& Planets IV, eds. V. Mannings, A. P. Boss, \& S. S. Russell (Tucson: The University of Arizona Press), 97

Wolfire, M. G., \& Cassinelli, J. 1987, ApJ, 319, 850

Wright, M. C. H., Sandell, G., Wilner, D. J., \& Plambeck, R. L. 1992, ApJ, 393, 225

Wyrowski, F., Schilke, P., Walmsley, C. M., \& Menten, K. M. 1999, ApJ, 514, L43

Zweibel, E. G., \& McKee, C. F. 1995, ApJ, 439, 779 